\title{
Well-Posedness and Stability Result of the Nonlinear Thermodiffusion Full von Kármán Beam with Thermal Effect and Time-Varying Delay
}

\author{
Abdelbaki Choucha, ${ }^{1}$ Djamel Ouchenane, ${ }^{2}$ Salah Mahmoud Boulaaras $\mathbb{D},{ }^{3,4}$ \\ Bahri Belkacem Cherif $\mathbb{D D}^{3,5}$ and Mohamed Abdalla $\mathbb{D D}^{6,7}$
}

${ }^{1}$ Laboratory of Operator Theory and PDEs: Foundations and Applications, Department of Mathematics, Faculty of Exact Sciences, University of El Oued, Algeria

${ }^{2}$ Laboratory of Pure and Applied Mathematics, Amar Telidji Laghouat University, Algeria

${ }^{3}$ Department of Mathematics, College of Sciences and Arts, Qassim University, Ar Rass, Saudi Arabia

${ }^{4}$ Laboratory of Fundamental and Applied Mathematics of Oran (LMFAO), University of Oran 1, Ahmed Benbella, Algeria

${ }^{5}$ Preparatory Institute for Engineering Studies in Sfax, Tunisia

${ }^{6}$ Mathematics Department, College of Science, King Khalid University, Abha 61413, Saudi Arabia

${ }^{7}$ Mathematics Department, Faculty of Science, South Valley University, Qena 83523, Egypt

Correspondence should be addressed to Salah Mahmoud Boulaaras; s.boularas@qu.edu.sa, Bahri Belkacem Cherif; bahi1968@yahoo.com, and Mohamed Abdalla; moabdalla@kku.edu.sa

Received 5 March 2021; Revised 19 March 2021; Accepted 22 March 2021; Published 5 April 2021

Academic Editor: Qifeng Zhang

Copyright (c) 2021 Abdelbaki Choucha et al. This is an open access article distributed under the Creative Commons Attribution License, which permits unrestricted use, distribution, and reproduction in any medium, provided the original work is properly cited.

In this work, we consider a new full von Kármán beam model with thermal and mass diffusion effects according to the GurtinPinkin model combined with time-varying delay. Heat and mass exchange with the environment during thermodiffusion in the von Kármán beam. We establish the well-posedness and the exponential stability of the system by the energy method under suitable conditions.

\section{Introduction and Preliminaries}

In this paper, we are concerned with the following problem:

$$
\left\{\begin{array}{l}
w_{t t}-d_{1}\left[\left(u_{x}+\frac{1}{2}\left(w_{x}\right)^{2}\right) w_{x}\right]_{x}+d_{2} w_{x x x x}+\mu_{1} w_{t}+\mu_{2} w_{t}(x, t-\tau(t))=0 \\
u_{t t}-d_{1}\left[u_{x}+\frac{1}{2}\left(w_{x}\right)^{2}\right]_{x}-\delta_{1} \theta_{x}-\delta_{2} P_{x}=0 \\
c \theta_{t}+d P_{t}-\int_{0}^{\infty} \beta_{1}(\sigma) \theta_{x x}(t-\sigma) d \sigma-\delta_{1} u_{t x}=0 \\
d \theta_{t}+r P_{t}-\int_{0}^{\infty} \beta_{2}(\sigma) P_{x x}(t-\sigma) d \sigma-\delta_{2} u_{t x}=0
\end{array}\right.
$$

where

$$
(x, \sigma, t) \in(0, L) \times \mathbb{R}_{+} \times(0, \infty) .
$$

Here, $\tau(t)>0$ represents the time-varying delay, and $d_{1}$, $d_{2}, \delta_{1}, \delta_{2}, c, d, r$, and $\mu_{1}$ are positive constants; $\mu_{2}$ is a real number, and $\beta_{1}$ and $\beta_{2}$ are the relaxation functions, with the initial data

$$
\begin{gathered}
w(x, 0)=w_{0}(x), \\
w_{t}(x, 0)=w_{1}(x), \\
u(x, 0)=u_{0}(x),
\end{gathered}
$$




$$
\begin{gathered}
u_{t}(x, 0)=u_{1}(x), \\
\theta(x, 0)=\theta_{0}(x), \\
P(x, 0)=P_{0}(x), \\
w_{t}(x, t-\tau(0))=f_{0}(x, t-\tau(0)),
\end{gathered}
$$

where

$$
(x, t) \in(0, L) \times(0, \tau(0)),
$$

and Neumann-Dirichlet boundary conditions

$$
\begin{aligned}
w(x, t) & =u(x, t)=P(x, t)=0, \quad x=0, L, \forall t \geq 0, \\
w_{x}(x, t) & =\theta(x, t)=0, \quad x=0, L, \forall t \geq 0 .
\end{aligned}
$$

The case of time-varying delay in the wave equation has been studied recently by Nicaise et al. [1]; they proved the exponential stability under the condition

$$
\mu_{2}<\sqrt{1-d} \mu_{1}
$$

where $d$ is a constant that satisfies

$$
\tau^{\prime}(t) \leq d<1, \quad \forall t>0
$$

For the wave equation with a time-varying delay, in [1], the authors consider the system

$$
\left\{\begin{array}{l}
u_{t t}-\Delta u=0, \\
u(x, t)=0 \\
\frac{d u}{d v}(x, t)=\mu_{1} u_{t}(x, t)+\mu_{2} u_{t}(x, t-\tau(t)),
\end{array}\right.
$$

where the time-varying delay $\tau(t)>0$ satisfies

$$
\begin{aligned}
0 & \leq \tau(t) \leq \bar{\tau}, \quad \forall t>0, \\
\tau^{\prime}(t) & \leq 1, \quad \forall t>0, \\
\tau(t) & \in W^{2, \infty}([0, T]), \quad \forall T>0 .
\end{aligned}
$$

They proved the exponential stability under suitable conditions.

The purpose of this work is to study problem (1)-(5), with a delay term appearing in the control term at the first equation, introducing the time-varying delay term $\beta_{2} w_{t}(x, t-\tau(t))$; thermal and mass diffusion effects make the problem different from those considered in the literature (see [2-30]).

This paper is organized as follows: in the rest of this section, we put the preliminaries necessary for problem (1); in Section 2, we establish the well-posedness. As for Section 3, we prove the exponential stability result by the energy method and Lyapunov function.
In order to prove the existence of a unique solution of problem (1)-(5), we introduce the new variable

$$
z(x, \rho, t)=w_{t}(x, t-\tau(t) \rho)
$$

Then, we obtain

$$
\left\{\begin{array}{l}
\tau(t) z_{t}(x, \rho, t)+\left(1-\tau^{\prime}(t) \rho\right) z_{\rho}(x, \rho, t)=0, \\
z(x, 0, t)=w_{t}(x, t) .
\end{array}\right.
$$

And it is more convenient to work in the history space setting by introducing the so-called summed past history of $\theta$ and $P$ defined by (see [31-36])

$$
\left\{\begin{array}{l}
\eta^{t}(\sigma)=\int_{0}^{\sigma} \theta(t-\zeta) d \zeta \\
v^{t}(\sigma)=\int_{0}^{\sigma} P(t-\zeta) d \zeta, \quad(t, \sigma) \in[0, \infty) \times \mathbb{R}_{+} .
\end{array}\right.
$$

Differentiating $(14)_{1}$ and $(14)_{2}$, we get

$$
\left\{\begin{array}{l}
\eta_{t}^{t}(\sigma)+\eta_{\sigma}^{t}(\sigma)=\theta(t) \\
v_{t}^{t}(\sigma)+v_{\sigma}^{t}(\sigma)=P(t)
\end{array}\right.
$$

with the boundary and initial conditions

$$
\begin{aligned}
& \eta^{t}(0)=v^{t}(0)=0, \quad t \geq 0, \\
& \eta^{0}(\sigma)=\eta_{0}(\sigma), v^{0}(\sigma)=v_{0}(\sigma), \quad \sigma \geq 0 .
\end{aligned}
$$

We set

$$
\left\{\begin{array}{l}
\eta_{0}(\sigma)=\int_{0}^{\sigma} \bar{\theta}_{0}(\tau) d \tau \\
\nu_{0}(\sigma)=\int_{0}^{\sigma} \bar{P}_{0}(\tau) d \tau, \sigma \in \mathbb{R}_{+} .
\end{array}\right.
$$

Concerning the memory kernels $\beta_{1}$ and $\beta_{2}$, we set

$$
\begin{aligned}
& \beta(\sigma)=-\beta_{1}{ }^{\prime}(\sigma), \\
& \lambda(\sigma)=-\beta_{2}{ }^{\prime}(\sigma) .
\end{aligned}
$$

Assuming $\beta_{1}(\infty)=\beta_{2}(\infty)=0$, then from (14), we infer

$$
\left\{\begin{array}{l}
\int_{0}^{\infty} \beta_{1}(\sigma) \theta(t-\sigma) d \sigma=-\int_{0}^{\infty} \beta_{1}{ }^{\prime}(\sigma) \eta^{t}(\sigma) d \sigma, \\
\int_{0}^{\infty} \beta_{2}(\sigma) P(t-\sigma) d \sigma=-\int_{0}^{\infty} \beta_{2}{ }^{\prime}(\sigma) \nu^{t}(\sigma) d \sigma,
\end{array}\right.
$$


and therefore,

$$
\left\{\begin{array}{l}
\int_{0}^{\infty} \beta_{1}(\sigma) \theta_{x x}(t-\sigma) d \sigma=\int_{0}^{\infty} \beta(\sigma) \eta_{x x}^{t}(\sigma) d \sigma \\
\int_{0}^{\infty} \beta_{2}(\sigma) P_{x x}(t-\sigma) d \sigma=\int_{0}^{\infty} \lambda(\sigma) v^{t}(\sigma) d \sigma .
\end{array}\right.
$$

Consequently, the problem is equivalent to

$$
\left\{\begin{array}{l}
w_{t t}-d_{1}\left[\left(u_{x}+\frac{1}{2}\left(w_{x}\right)^{2}\right) w_{x}\right]_{x}+d_{2} w_{x x x x}+\mu_{1} w_{t}+\mu_{2} z(x, 1, t)=0 \\
u_{t t}-d_{1}\left[u_{x}+\frac{1}{2}\left(w_{x}\right)^{2}\right]_{x}-\delta_{1} \theta_{x}-\delta_{2} P_{x}=0 \\
c \theta_{t}+d P_{t}-\int_{0}^{\infty} \beta(\sigma) \eta_{x x}^{t}(\sigma) d \sigma-\delta_{1} u_{t x}=0 \\
d \theta_{t}+r P_{t}-\int_{0}^{\infty} \lambda(\sigma) v_{x x}^{t}(\sigma) d \sigma-\delta_{2} u_{t x}=0 \\
\eta_{t}^{t}+\eta_{\sigma}^{t}=\theta \\
v_{t}^{t}+v_{\sigma}^{t}=P \\
\tau(t) z_{t}(x, \rho, t)+\left(1-\tau^{\prime}(t) \rho\right) z_{\rho}(x, \rho, t)=0
\end{array}\right.
$$

where

$$
(x, \sigma, \rho, t) \in(0, L) \times \mathbb{R}_{+} \times(0,1) \times(0, \infty),
$$

with the initial and boundary conditions

$$
\left\{\begin{array}{l}
w(x, t)=w_{x}(x, t)=u(x, t)=P(x, t)=\theta(x, t)=0, \quad x=0, L, \\
w(x, 0)=w_{0}(x), w_{t}(x, 0)=w_{1}(x), \\
u(x, 0)=u_{0}(x), u_{t}(x, 0)=u_{1}(x), \\
\theta(x, 0)=\theta_{0}(x), P(x, 0)=P_{0}(x), \\
z(x, \rho, 0)=f_{0}(x,-\rho \tau(0)), \\
\eta^{0}(x, \sigma)=\eta_{0}(x, \sigma), v^{0}(x, \sigma)=v_{0}(x, \sigma), \quad(x, \sigma) \in(0,1) \times \mathbb{R}_{+},
\end{array}\right.
$$

$$
\forall(x, \rho, \sigma, t) \in(0, L) \times(0,1) \times(0, \infty) \times(0, \infty),
$$

where the function $\tau(t)$ satisfies (7), (11), and the condition

$$
0<\tau_{0}<\tau(t)<\bar{\tau}, \quad \forall t>0 .
$$

In this paper, we establish the well-posedness and prove the exponential stability by using the variable of Kato under some restrictions and assumptions:

(H1).

$$
\left|\mu_{2}\right| \leq \sqrt{1-d} \mu_{1}
$$

(H2). The symmetric matrix $\Lambda$ is positive definite, where

$$
\Lambda=\left(\begin{array}{l}
c d \\
d r
\end{array}\right) .
$$

That is, $|\Lambda|=c r-d^{2}>0$ implies that

$$
c \int_{0}^{L} \theta^{2} d x+2 d \int_{0}^{L} \theta P d x+r \int_{0}^{L} P^{2} d x>0 .
$$

Condition (28) is needed to stabilize the system when diffusion effects are added to thermal effects (see, e.g., [31-38] for more information on this). By virtue of $c r>d^{2}$, we deduce that $d / c<r / d$. Let, then, $\zeta$ be a number chosen in such a way that

$$
\frac{d}{c}<\zeta<\frac{r}{d}
$$

Thus, Young's inequality leads to

$$
2 d \int_{0}^{L} \theta P d x \leq \frac{d}{\zeta} \int_{0}^{L} \theta^{2} d x+d \zeta \int_{0}^{L} P^{2} d x
$$

(H3). We assume the following set of hypotheses on $\mu$ and $\lambda$ :

$$
\beta, \lambda \in C^{1}\left(\mathbb{R}_{+}\right) \cap L^{1}\left(\mathbb{R}_{+}\right),
$$

$\beta(\sigma), \lambda(\sigma) \geq 0, \beta^{\prime}(\sigma), \lambda^{\prime}(\sigma) \leq 0, \quad \forall \sigma \in \mathbb{R}_{+}$,

$\beta^{\prime}(\sigma)+\alpha_{1} \beta(\sigma) \leq 0, \lambda^{\prime}(\sigma)+\alpha_{2} \lambda(\sigma) \leq 0, \quad$ for some $\alpha_{1}, \alpha_{2}>0, \forall \sigma \in \mathbb{R}_{+}$,

$$
\left\{\begin{array}{l}
\beta(0)=\int_{0}^{\infty} \beta(\sigma) d \sigma:=\beta_{0}>0 \\
\lambda(0)=\int_{0}^{\infty} \lambda(\sigma) d \sigma:=\lambda_{0}>0
\end{array}\right.
$$

Let $f$ be a memory kernel satisfying the assumptions (31) and (32).

Now, we consider the weighted Hilbert spaces

$$
\begin{aligned}
\mathscr{M}_{f} & =L^{2}\left(\mathbb{R}_{+}, H_{0}^{1}(0, L)\right) \\
& =\left\{\Phi: \mathbb{R}_{+} \rightarrow H_{0}^{1}(0, L) / \int_{0}^{L} \int_{0}^{\infty} f(\sigma) \Phi_{x}^{2}(\sigma) d \sigma d x<\infty\right\},
\end{aligned}
$$

equipped with the inner product

$$
<\Phi, \Psi>_{\mathscr{M}_{f}}=\int_{0}^{L} \int_{0}^{\infty} f(\sigma) \Phi_{x}(\sigma) \Psi_{x}(\sigma) d \sigma d x,
$$


and the norm

$$
\|\Phi\|_{\mathscr{M}_{f}}^{2}=\langle\Phi, \Phi\rangle_{\mathscr{M}_{f}}=\int_{0}^{L} \int_{0}^{\infty} f(\sigma) \Phi_{x}^{2}(\sigma) d \sigma d x
$$

We also introduce the linear operator $T$ on $\mathscr{M}_{f}$ defined by

$$
T_{\Phi}=-\Phi_{\sigma}, \quad \Phi \in \mathscr{D}(T),
$$

with

$$
\mathscr{D}(T)=\left\{\Phi \in \mathscr{M}_{f} / \Phi_{\sigma} \in \mathscr{M}_{f}, \Phi(0)=0\right\}
$$

where $\Phi_{\sigma}$ is the distributional derivative of $\Phi$ with respect to the internal variable $\sigma$, and then, the operator $T$ is the infinitesimal generator of a $C_{0}$-semigroup of contractions. Following Ref. [39], there holds

$$
\begin{aligned}
\langle T \Phi, \Phi\rangle_{\mathscr{M}_{f}} & =\left\langle-\Phi_{\sigma}, \Phi\right\rangle_{\mathscr{M}_{f}} \\
& =-\frac{1}{2} \int_{0}^{\infty} f(\sigma) \frac{d}{d \sigma} \int_{0}^{L} \Phi_{x}^{2}(\sigma) d x d \sigma, \quad \forall \Phi \in \mathscr{D}(T) .
\end{aligned}
$$

Integration by parts yields

$$
\begin{aligned}
& \int_{0}^{\infty} f(\sigma) \frac{d}{d \sigma} \int_{0}^{L} \Phi_{x}^{2}(\sigma) d x d \sigma \\
& \quad=\left.f(\sigma) \int_{0}^{L} \Phi_{x}^{2}(\sigma) d x\right|_{0} ^{\infty}-\int_{0}^{\infty} f^{\prime}(\sigma) \int_{0}^{L} \Phi_{x}^{2}(\sigma) d x d \sigma .
\end{aligned}
$$

Hence, from (31), we obtain

$$
<T \Phi, \Phi>_{\mathscr{M}_{f}}=\frac{1}{2} \int_{0}^{\infty} f^{\prime}(\sigma) \int_{0}^{L} \Phi_{x}^{2}(\sigma) d x d \sigma \leq 0 .
$$

As a direct consequence, we deduce from (32) and (40) that

$$
\begin{aligned}
<T \eta, \eta\rangle_{\mathscr{M}_{\beta}} & =\frac{1}{2} \int_{0}^{\infty} \beta^{\prime}(\sigma) \int_{0}^{L} \eta_{x}^{2}(\sigma) d x d \sigma \\
& \leq-\frac{\alpha_{1}}{2} \int_{0}^{\infty} \beta(\sigma) \int_{0}^{L} \eta_{x}^{2}(\sigma) d x d \sigma=-\frac{\alpha_{1}}{2}\left\|\eta_{x}\right\|_{\mathscr{M}_{\beta}}^{2}, \\
<T \nu, v>_{\mathscr{M}_{\lambda}} & =\frac{1}{2} \int_{0}^{\infty} \lambda^{\prime}(\sigma) \int_{0}^{L} v_{x}^{2}(\sigma) d x d \sigma \\
& \leq-\frac{\alpha_{2}}{2} \int_{0}^{\infty} \lambda(\sigma) \int_{0}^{L} v_{x}^{2}(\sigma) d x d \sigma=-\frac{\alpha_{2}}{2}\left\|v_{x}\right\|_{\mathscr{M}_{\lambda}}^{2}
\end{aligned}
$$

for all $\eta, v \in \mathscr{D}(T)$. Finally, we define the operator $\mathbb{L}_{f}$ : $\mathscr{D}\left(\mathbb{L}_{f}\right) \rightarrow L^{2}(0, L)$ by

$$
\mathbb{L}_{f} \Phi=\int_{0}^{\infty} f(\sigma) \Phi_{x x}(\sigma) d \sigma
$$

with the domain

$$
\mathscr{D}\left(\mathbb{L}_{f}\right)=\left\{\Phi \in \mathscr{M}_{f} / \int_{0}^{\infty} f(\sigma) \Phi_{x x}(\sigma) d \sigma \in L^{2}(0, L), \Phi(0)=0\right\} .
$$

\section{Well-Posedness}

In this section, we give sufficient conditions that guarantee the well-posedness of this problem. Let

$$
U=\left(w, w_{t}, u, u_{t}, \theta, \eta^{t}, P, v^{t}, z\right)^{T}
$$

For the sake of simplicity, we write $\eta=\eta^{t}(\sigma)$ and $v=$ $v^{t}(\sigma)$ and the new dependent variables $\varphi=\omega_{t}$ and $\psi=u_{t}$; then, (21)-(23) can be written as

$$
\left\{\begin{array}{l}
U^{\prime}=\mathscr{A}(t) U+\mathscr{F}(U), \\
U(0)=\left(w_{0}, w_{1}, u_{0}, u_{1}, \theta_{0}, \eta_{0}, P_{0}, v_{0}, f_{0}(.,-\rho \tau(0))\right)^{T},
\end{array}\right.
$$

with the linear problem

$$
\left\{\begin{array}{l}
U^{\prime}=\mathscr{A}(t) U, \\
U(0)=\left(w_{0}, w_{1}, u_{0}, u_{1}, \theta_{0}, \eta_{0}, P_{0}, v_{0}, f_{0}(.,-\rho \tau(0))\right)^{T},
\end{array}\right.
$$

where the time-varying operator $\mathscr{A}$ is defined by

$$
\mathscr{A}(t)\left(\begin{array}{c}
w \\
\varphi \\
u \\
\psi \\
\eta \\
\eta \\
P \\
v \\
z
\end{array}\right)=\left(\begin{array}{c}
\varphi \\
-d_{2} w_{x x x x}-\mu_{1} \varphi-\mu_{2} z(x, 1, t) \\
\psi \\
d_{1} u_{x x}+\delta_{1} \theta_{x}+\delta_{2} P_{x} \\
-\frac{1}{\alpha_{1}}\left[\left(d \delta_{2}-r \delta_{1}\right) \psi_{x}-r \mathbb{L}_{\beta} \eta+d \mathbb{L}_{\lambda} v\right] \\
\theta+T \eta \\
-\frac{1}{\alpha_{2}}\left[\left(d \delta_{1}-c \delta_{2}\right) \psi_{x}+d \mathbb{L}_{\beta} \eta-c \mathbb{L}_{\lambda} v\right] \\
P+T v \\
\frac{\left(\tau^{\prime}(t) \rho-1\right)}{\tau(t)} z_{\rho}
\end{array}\right),
$$




$$
\mathscr{F}(U)=\left(\begin{array}{c}
0 \\
d_{1}\left[u_{x}+\frac{1}{2}\left(w_{x}\right)^{2}\right]_{x} \\
0 \\
\frac{d_{1}}{2}\left(w_{x}\right)^{2} \\
0 \\
0 \\
0 \\
0 \\
0
\end{array}\right) .
$$

The energy space $\mathscr{H}$ is defined as

$$
\begin{aligned}
\mathscr{H}= & {\left[H^{4}(0, L) \cap H_{0}^{2}(0, L)\right] \times H_{0}^{1}(0, L) } \\
& \times\left[H^{2}(0, L) \cap H_{0}^{2}(0, L)\right] \times H_{0}^{1}(0, L) \times L^{2}(0, L) \\
& \times \mathscr{M}_{\beta} \times L^{2}(0, L) \times \mathscr{M}_{\lambda} \times L^{2}((0, L),(0,1)),
\end{aligned}
$$

and the domain of $\mathscr{A}$ is

$$
\begin{aligned}
\mathscr{D}(\mathscr{A}(t))= & \left\{U \in \mathscr{H} / \varphi=z(., 0), \theta, P \in H_{0}^{1}(0, L), \mathbb{L}_{\beta} \eta, \mathbb{L}_{\lambda} v\right. \\
& \left.\in L^{2}(0, L), \eta, v \in \mathscr{D}(T)\right\} .
\end{aligned}
$$

We equip $\mathscr{H}$ with the inner product

$$
\begin{aligned}
<U, \bar{U}>_{\mathscr{H}}= & \int_{0}^{L}\left\{\varphi \bar{\varphi}+d_{1} u_{x} \bar{u}_{x}+\psi \bar{\psi}+d_{2} w_{x x} \bar{w}_{x x}\right\} d x \\
& +\int_{0}^{L} \int_{0}^{1} z(x, \rho, t) \bar{z}(x, \rho, t) d \rho d x \\
& \left.+<\Lambda(\theta, P)^{T},(\bar{\theta}, \bar{P})^{T}>+<\eta, \bar{\eta}>_{\mathscr{M}_{\beta}}+<v, \bar{v}\right\rangle_{\mathscr{M}_{\lambda}},
\end{aligned}
$$

with the existence and the uniqueness in the following result.

Theorem 1. Let (7), (11), and (25) be satisfied and assume that (26)-(31) hold. Then, for all $U_{0} \in \mathscr{D}(\mathscr{A}(0))$, there exists a unique solution $U$ of problem (21)-(23) satisfying

$$
U \in C\left([0,+\infty), \mathscr{D}(\mathscr{A}(0)) \cap C^{1}([0,+\infty), \mathscr{H}) .\right.
$$

In order to prove Theorem 1, we will use the variable norm technique developed by Kato in [40]. The following theorem is proved in [40].

Theorem 2. Assume that

(1) $\mathscr{D}(\mathscr{A}(0))$ is a dense subset of $\mathscr{H}$

(2) $\mathscr{D}(\mathscr{A}(t))=\mathscr{D}(\mathscr{A}(0)), \forall t>0$
(3) For all $t \in[0, T], \mathscr{A}(t)$ generates a strongly continuous semigroup on $\mathscr{H}$ and the family $\mathscr{A}=\{\mathscr{A}(t): t \in[0, T]\}$ is stable with stability constants $C$ and $m$ independent of $t$; i.e., the semigroup $\left(S_{t}(s)\right)_{s \geq 0}$ generated by $\mathscr{A}(t)$ satisfies

$$
\left\|S_{t}(s)(u)\right\|_{\mathscr{H}} \leq C e^{m s}\|u\|_{\mathscr{H}}, \quad \forall u \in \mathscr{H}, s \geq 0 .
$$

(4) $d_{t} \mathscr{A}(t) \in L_{*}^{\infty}([0, T], B(\mathscr{D}(\mathscr{A}(0)), \mathscr{H}))$, where $L_{*}^{\infty}([0$, $T], B(\mathscr{D}(\mathscr{A}(0)), \mathscr{H}))$ is the space of equivalent classes of essentially bounded, strongly measurable functions from $[0, T]$ into the set $B(\mathscr{D}(\mathscr{A}(0)), \mathscr{H})$ of bounded operators from $\mathscr{D}(\mathscr{A}(0))$ into $\mathscr{H}$

Then, problem (46) has a unique solution

$$
U \in C([0, T], \mathscr{D}(\mathscr{A}(0))) \cap C^{1}([0, T], \mathscr{H}),
$$

for any initial datum in $\mathscr{D}(\mathscr{A}(0))$.

Proof. To prove Theorem 1, we use the method in [1] with the necessary modification.

(1) First, we show that $\mathscr{D}(\mathscr{A}(0))$ is dense in $\mathscr{H}$

Let $F=\left(f_{1}, f_{2}, f_{3}, f_{4}, f_{5}, f_{6}, f_{7}, f_{8}, f_{9}\right) \in \mathscr{H}$ be orthogonal to all elements of $\mathscr{D}(\mathscr{A}(0))$ with respect to the inner product $\langle., .\rangle_{\mathscr{H}}$ :

$$
\begin{aligned}
0= & <U, F>_{\mathscr{H}}=\int_{0}^{L}\left\{\varphi f_{2}+\psi f_{4}+d_{2} w_{x x} f_{1 x x}+d_{1} u_{x} f_{3 x}\right\} d x \\
& +\int_{0}^{L} \int_{0}^{1} z(x, \rho, t) f_{9} d \rho d x+<\Lambda(\theta, P)^{T},\left(f_{5}, f_{7}\right)^{T}> \\
& +<\eta, f_{6}>\mathscr{M}_{\beta}+<\nu, f_{8}>\mathscr{M}_{\lambda} .
\end{aligned}
$$

For all $U=(w, \varphi, u, \psi, \theta, \eta, P, v, z)^{T} \in \mathscr{D}(\mathscr{A}(0))$, our goal is to prove that $f_{i}=0, \forall i=1, \cdots, 9$. Let us first take $z \in \mathscr{D}((0, L) \times(0,1))$ and $w=\varphi=\psi=u=\theta=q=\phi=0$, so the vector $U=(0,0,0,0,0,0,0,0, z)^{T} \in \mathscr{D}(\mathscr{A}(0))$, and therefore, from (55), we deduce that

$$
\int_{0}^{L} \int_{0}^{1} z(x, \rho) f_{7} d \rho d x=0
$$

Since $\mathscr{D}((0, L) \times(0,1))$ is dense in $L^{2}((0, L) \times(0,1))$, it follows then that $f_{9}=0$.

Similarly, let $\varphi \in H_{0}^{1}(0, L)$; then, $U=(0, \varphi, 0,0,0,0,0$, $0,0)^{T} \in \mathscr{D}(\mathscr{A}(0))$, which implies from (55) that

$$
\int_{0}^{L} \varphi f_{2} d x=0
$$

So, as above, $f_{2}=0$. 
And let $U=(w, 0,0,0,0,0,0,0,0)^{T}$; then, we obtain from (55) that

$$
\int_{0}^{L} w_{x x} f_{1 x x} d x=0
$$

It is obvious that $U=(w, 0,0,0,0,0,0,0,0)^{T} \in \mathscr{D}(\mathscr{A}(0))$ only if $w \in H^{4}(0, L) \cap H_{0}^{2}(0, L)$ is dense in $H_{0}^{2}(0, L)$, with respect to the inner product

$$
<g, h>_{H_{0}^{2}(0, L)} \int_{0}^{L} g_{x x} h_{x x} d x .
$$

We get $f_{1}=0$. By the same ideas as above, we can also show that $f_{3}=0$.

For $u \in \mathscr{D}(\mathscr{A}(t))$, we get from (55) that

$$
\int_{0}^{L} u_{x} f_{3 x} d x=0
$$

and by the density of $\mathscr{D}(\mathscr{A}(t))$ in $H_{0}^{1}(0, L)$, we obtain $f_{3}=0$.

For $\psi \in \mathscr{D}(\mathscr{A}(t))$, we get from (55) that

$$
\int_{0}^{L} \psi f_{4} d x=0
$$

and by the density of $\mathscr{D}(\mathscr{A}(t))$ in $H^{1}(0, L)$, we obtain $f_{4}=0$.

Next, let $U=(0,0,0,0, \theta, 0,0,0,0)^{T}$; then, we obtain from (55) that

$$
\int_{0}^{L} \theta f_{5} d x=0
$$

It is obvious that $U=(0,0,0,0, \theta, 0,0,0,0)^{T} \in \mathscr{D}(\mathscr{A}(0))$ only if $\theta \in L^{2}(0, L)$ is dense in $L^{2}(0, L)$; we get $f_{5}=0$; for $\eta \in \mathscr{M}_{\beta}$, we get from (55) that

$$
\int_{0}^{L} \int_{0}^{\infty} \beta(\sigma) \eta_{x} f_{6 x} d \sigma d x=0
$$

which gives $f_{6}=0$. Similarly, for $P$ and $v$. This completes the proof of (1).

(2) With our choice, $\mathscr{D}(\mathscr{A}(t))$ is independent of $t$; consequently,

$$
\mathscr{D}(\mathscr{A}(t))=\mathscr{D}(\mathscr{A}(0)), \quad \forall t>0 .
$$

(3) Now, we show that the operator $\mathscr{A}(t)$ generates a $C_{0}$-semigroup in $\mathscr{H}$ for a fixed $t$. We define the time-dependent inner product on $\mathscr{H}$ :

$$
\begin{aligned}
<U, \bar{U}>_{t}= & \int_{0}^{L}\left\{\varphi \bar{\varphi}+d_{1} u_{x} \bar{u}_{x}+\psi \bar{\psi}+d_{2} w_{x x} \bar{w}_{x x}\right\} d x \\
& +\xi \tau(t) \int_{0}^{L} \int_{0}^{1} z(x, \rho, t) \bar{z}(x, \rho, t) d \rho d x \\
& +<\Lambda(\theta, P)^{T},(\bar{\theta}, \bar{P})^{T}>+<\eta, \bar{\eta}>_{\mathscr{M}_{\beta}}+<v, \bar{v}>_{\mathscr{M}_{\lambda}},
\end{aligned}
$$

where $\xi$ satisfies

$$
\frac{\left|\mu_{2}\right|}{\sqrt{1-d}} \leq \xi \leq\left(2 \mu_{1}-\frac{\left|\mu_{2}\right|}{\sqrt{1-d}}\right)
$$

thanks to hypothesis (26).

Let us set

$$
\kappa(t)=\frac{\left(\tau^{\prime}(t)^{2}+1\right)^{1 / 2}}{2 \tau(t)} .
$$

In this step, we prove the dissipativity of the operator $\overline{\mathscr{A}}(t)=\mathscr{A}(t)-\tau(t) I$.

For a fixed $t$ and $U=(w, \varphi, u, \psi, \theta, \eta, P, v, z)^{T} \in \mathscr{D}(\mathscr{A}(t))$, we have

$$
\begin{aligned}
<\mathscr{A}(t) U, U>_{t}= & -\mu_{1} \int_{0}^{L} \varphi^{2} d x-\mu_{2} \int_{0}^{L} \varphi z(x, 1, t) d x \\
& +<T \eta, \eta>_{\mathscr{M}_{\beta}}+<T v, \eta>_{\mathscr{M}_{\lambda}} \\
& -\xi \int_{0}^{L} \int_{0}^{1}\left(1-\tau^{\prime}(t) \rho\right) z(x, \rho, t) z_{\rho}(x, \rho, t) d \rho d x .
\end{aligned}
$$

Observe that

$$
\begin{aligned}
\int_{0}^{L} \int_{0}^{1}\left(1-\tau^{\prime}(t) \rho\right) z(x, \rho, t) z_{\rho}(x, \rho, t) d \rho d x \\
=\frac{1}{2} \int_{0}^{L} \int_{0}^{1}\left(1-\tau^{\prime}(t) \rho\right) \frac{d}{d \rho} z^{2} d \rho d x \\
=\frac{\tau^{\prime}(t)}{2} \int_{0}^{L} \int_{0}^{1} z^{2}(x, \rho, t) d \rho d x \\
\quad+\frac{1}{2} \int_{0}^{L}\left\{z^{2}(x, 1, t)\left(1-\tau^{\prime}(t)\right)-z^{2}(x, 0, t)\right\} d \rho d x, \\
<T \eta, \eta>\mathscr{M}_{\beta}+<T v, \eta>_{\mathscr{M}_{\lambda}} \\
=+\frac{1}{2} \int_{0}^{\infty} \beta^{\prime}(\sigma) \int_{0}^{L} \eta_{x}^{2}(\sigma) d x d \sigma+\frac{1}{2} \int_{0}^{\infty} \lambda^{\prime}(\sigma) \int_{0}^{L} v_{x}^{2}(\sigma) d x d \sigma \\
\leq-\frac{\alpha_{1}}{2}\|\eta(\sigma)\|_{\mathscr{M}_{\beta}}^{2}-\frac{\alpha_{2}}{2}\|v(\sigma)\|_{\mathscr{M}_{\lambda},}^{2}
\end{aligned}
$$


whereupon

$$
\begin{aligned}
<\mathscr{A}(t) U, U>_{t}= & -\mu_{1} \int_{0}^{L} \varphi^{2} d x-\mu_{2} \int_{0}^{L} \varphi z(x, 1, t) d x \\
& -\frac{\alpha_{1}}{2}\|\eta(\sigma)\|_{\mathscr{M}_{\beta}}^{2}-\frac{\alpha_{2}}{2}\|v(\sigma)\|_{\mathscr{M}_{\lambda}}^{2} \\
& -\frac{\xi \tau^{\prime}(t)}{2} \int_{0}^{L} \int_{0}^{1} z^{2}(x, \rho, t) d \rho d x \\
& -\frac{\xi}{2} \int_{0}^{L} z^{2}(x, 1, t)\left(1-\tau^{\prime}(t)\right) d x+\frac{\xi}{2} \int_{0}^{L} \varphi^{2} d x .
\end{aligned}
$$

By using Young's inequality and (7), we get

$$
\begin{aligned}
<\mathscr{A}(t) U, U>_{t} \leq & \left(-\mu_{1}+\frac{\left|\mu_{2}\right|}{2 \sqrt{1-d}}+\frac{\xi}{2}\right) \int_{0}^{L} \varphi^{2} d x \\
& +\left(\frac{\left|\mu_{2}\right| \sqrt{1-d}}{2}-\xi \frac{(1-d)}{2}\right) \int_{0}^{L} z^{2}(x, 1, t) d x \\
& \left.-\frac{\alpha_{1}}{2}\|\eta(\sigma)\|_{\mathscr{M}_{\beta}}^{2}-\frac{\alpha_{2}}{2}\|v(\sigma)\|_{\mathscr{M}_{\lambda}}^{2}+\kappa(t)<U, U\right\rangle_{t},
\end{aligned}
$$

under condition (66) which allows to write

$$
\begin{aligned}
-\mu_{1}+\frac{\left|\mu_{2}\right|}{2 \sqrt{1-d}}+\frac{\xi}{2} & \leq 0, \\
\frac{\left|\mu_{2}\right| \sqrt{1-d}}{2}-\xi \frac{(1-d)}{2} & \leq 0 .
\end{aligned}
$$

Consequently, the operator $\quad \bar{A}(t)=\mathscr{A}(t)-\kappa(t) I \quad$ is dissipative.
Now, we prove the subjectivity of the operator $I-\mathscr{A}(t)$ for fixed $t>0$.

Let $\left(f_{1}, f_{2}, f_{3}, f_{4}, f_{5}, f_{6}, f_{7}, f_{8}, f_{9}\right)^{T} \in \mathscr{H}$; we seek $U=$ $(w, \varphi, u, \psi, \theta, \eta, P, v, z)^{T} \in \mathscr{D}(\mathscr{A}(t))$ solution of the following system:

$$
\left\{\begin{array}{l}
w-\varphi=f_{1}, \\
\varphi+d_{2} w_{x x x x}+\mu_{1} \varphi+\mu_{2} z(., 1, t)=f_{2}, \\
u-\psi=f_{3}, \\
\psi-d_{1} u_{x x}-\delta_{1} \theta_{x}-\delta_{2} P_{x}=f_{4}, \\
\alpha_{1} \theta+\left(d \delta_{2}-r \delta_{1}\right) \psi_{x}-r \mathbb{L}_{\beta} \eta+d \mathbb{L}_{\lambda} v=\alpha_{1} f_{5}, \\
\eta-\theta-T \eta=f_{6}, \\
\alpha_{2} P+\left(d \delta_{1}-c \delta_{2}\right) \psi_{x}+d \mathbb{L}_{\beta} \eta+-c \mathbb{L}_{\lambda} v=\alpha_{2} f_{7}, \\
v-P-T v=f_{8}, \\
z-\frac{\left(\tau^{\prime}(t) \rho-1\right)}{\tau(t)} z_{\rho}=f_{9} .
\end{array}\right.
$$

Suppose that we have found $w$ and $u$. Then,

$$
\left\{\begin{array}{l}
w-\varphi=f_{1}, \\
u-\psi=f_{3} .
\end{array}\right.
$$

Furthermore, by (73), we can find $z$ as

$$
z(x, 0)=\varphi(x), \quad x \in(0, L) .
$$

Following the same approach as in [1], we obtain, by using the last equation in (73),

$$
\left\{\begin{array}{l}
z(x, \rho)=\varphi(x) e^{-\rho \tau(t)}+\tau(t) e^{-\rho \tau(t)} \int_{0}^{1} f_{9}(x, y) e^{y \tau(t)} d y, \quad \text { if } \tau^{\prime}(t)=0, \\
z(x, \rho)=\varphi(x) e^{\eta_{\rho}(t)}+e^{\eta_{\rho}(t)} \int_{0}^{1} \frac{\tau(t)}{1-\tau^{\prime}(t) y} f_{9}(x, y) e^{-\eta_{y}(t)} d y, \quad \text { if } \tau^{\prime}(t) \neq 0,
\end{array}\right.
$$

where $\eta_{y}(t)=\left(\tau(t) / \tau^{\prime}(t)\right) \ln \left(1-\tau^{\prime}(t) \rho\right)$. Whereupon, from

(74), we obtain

$$
\left\{\begin{array}{l}
z(x, \rho)=\varphi(x) e^{-\rho \tau(t)}-f_{1} e^{-\rho \tau(t)}+\tau(t) e^{-\rho \tau(t)} \int_{0}^{1} f_{9}(x, y) e^{y \tau(t)} d y, \quad \text { if } \tau^{\prime}(t)=0, \\
z(x, \rho)=\varphi(x) e^{\eta_{\rho}(t)}-f_{1} e^{\eta_{\rho}(t)}+e^{\eta_{\rho}(t)} \int_{0}^{1} \frac{\tau(t)}{1-\tau^{\prime}(t) y} f_{9}(x, y) e^{-\eta_{y}(t)} d y, \quad \text { if } \tau^{\prime}(t) \neq 0 .
\end{array}\right.
$$


Integrating $(73)_{6}$ and $(73)_{8}$ with $\eta(0)=v(0)=0$, we have

$$
\left\{\begin{array}{l}
\eta(\sigma)=\left(1-e^{-\sigma}\right) \theta+\int_{0}^{\sigma} e^{s-\sigma} f_{6}(s) d s \\
\nu(\sigma)=\left(1-e^{-\sigma}\right) P+\int_{0}^{\sigma} e^{s-\sigma} f_{8}(s) d s .
\end{array}\right.
$$

Substituting $(73)_{1,3,6,8,9}$ into the others, we obtain the following system. Now, we have to find $w, u, \theta$, and $P$ as solutions of the equations:

$$
\left\{\begin{array}{l}
w+d_{2} w_{x x x x}+\mu_{1} \varphi+\mu_{2} z(., 1, t)=f_{2}+f_{1}+\beta_{1} f_{1}, \\
u-d_{1} u_{x x}-\delta_{1} \theta_{x}-\delta_{2} P_{x}=f_{4}+f_{3}, \\
\alpha_{1} \theta-r C_{\beta} \theta_{x x}+d C_{\lambda} P_{x x}+\left(d \delta_{2}-r \delta_{1}\right) u_{x}=h_{3}, \\
\alpha_{2} P+d C_{\beta} \theta_{x x}-c C_{\lambda} P_{x x}+\left(d \delta_{1}-c \delta_{1}\right) u_{x}=h_{4} .
\end{array}\right.
$$

Solving (79), we get

$$
\left\{\begin{array}{l}
\mu_{3} w+d_{2} w_{x x x x}=h_{1}, \\
u-d_{1} u_{x x}-\delta_{1} \theta_{x}-\delta_{2} P_{x}=h_{2}, \\
\alpha_{1} \theta-r C_{\beta} \theta_{x x}+d C_{\lambda} P_{x x}+\left(d \delta_{2}-r \delta_{1}\right) u_{x}=h_{3}, \\
\alpha_{2} P+d C_{\beta} \theta_{x x}-c C_{\lambda} P_{x x}+\left(d \delta_{1}-c \delta_{1}\right) u_{x}=h_{4},
\end{array}\right.
$$

$$
\left\{\begin{array}{l}
\mu_{3}=1+\mu_{1}+e^{-\tau(t)}, \\
h_{1}=f_{2}+\left(1+\mu_{1}\right) f_{2}-\mu_{2} z_{0}, \\
h_{2}=f_{4}+f_{3}, \\
h_{3}=\alpha_{1} f_{5}+\left(d \delta_{2}-r \delta_{1}\right) f_{3 x}+r \int_{0}^{\infty} \beta(\sigma) \int_{0}^{\sigma} e^{s-\sigma} f_{6 x x}(s) d s d \sigma-d \int_{0}^{\infty} \lambda(\sigma) \int_{0}^{\sigma} e^{s-\sigma} f_{8 x x}(s) d s d \sigma, \\
h_{4}=\alpha_{2} f_{7}+\left(d \delta_{1}-c \delta_{2}\right) f_{5 x}-d \int_{0}^{\infty} \beta(\sigma) \int_{0}^{\sigma} e^{s-\sigma} f_{6 x x}(s) d s d \sigma+c \int_{0}^{\infty} \lambda(\sigma) \int_{0}^{\sigma-\sigma} f_{8 x x}(s) d s d \sigma .
\end{array}\right.
$$

From (77), we have

$$
z(x, 1)= \begin{cases}w(x) e^{-\tau(t)}+z_{0}(x), & \text { if } \tau^{\prime}(t)=0, \\ w(x) e^{\eta_{\rho}(t)}+z_{0}(x), & \text { if } \tau^{\prime}(t) \neq 0\end{cases}
$$

where $x \in(0, L)$ and

$z_{0}(x)=\left\{\begin{array}{l}-f_{1} e^{-\rho \tau(t)}+\tau(t) e^{-\rho \tau(t)} \int_{0}^{1} f_{9}(x, y) e^{y \tau(t)} d y, \quad \text { if } \tau^{\prime}(t)=0, \\ -f_{1} e^{\eta_{\rho}(t)}+e^{\eta_{\rho}(t)} \int_{0}^{1} \frac{\tau(t)}{1-\tau^{\prime}(t) y} f_{9}(x, y) e^{-\eta_{y}(t)} d y, \quad \text { if } \tau^{\prime}(t) \neq 0 .\end{array}\right.$

It is clear from the above formula that $z_{0}$ depends only on $f_{1}, f_{9}$. Consequently, problem (80) is equivalent to

$$
\zeta((w, u, \theta, P),(\widehat{w}, \widehat{u}, \widehat{\theta}, \widehat{P}))=\Gamma(\widehat{w}, \widehat{u}, \widehat{\theta}, \widehat{P})
$$

where the bilinear form $\zeta:\left[H_{0}^{2}(0, L) \times H_{0}^{1}(0, L) \times L^{2}(0, L) \times\right.$ $\left.L^{2}(0, L)\right]^{2} \rightarrow \mathbb{R}$ and the linear form $\Gamma:\left[H_{0}^{2}(0, L) \times H_{0}^{1}(0, L)\right.$ $\left.\times L^{2}(0, L) \times L^{2}(0, L)\right] \rightarrow \mathbb{R}$ are defined by

$$
\begin{aligned}
& \zeta((w, u, \theta, P),(\widehat{w}, \widehat{u}, \widehat{\theta}, \widehat{P})) \\
& =\int_{0}^{L}\left(\mu_{3} w \widehat{w}+d_{2} w_{x x} \widehat{w}_{x x}+u \widehat{u}+d_{1} u_{x} \widehat{u}_{x}\right) d x+\alpha_{1} \int_{0}^{L} \theta \widehat{\theta} d x \\
& \quad+\alpha_{2} \int_{0}^{L} P \widehat{P} d x+r C_{\beta} \int_{0}^{L} \theta_{x} \widehat{\theta}_{x} d x+c C_{\lambda} \int_{0}^{L} P_{x} \widehat{P}_{x} d x \\
& \quad-d C_{\beta} \int_{0}^{L} \theta_{x} \widehat{P}_{x} d x-d C_{\lambda} \int_{0}^{L} P_{x} \widehat{\theta}_{x} d x \\
& \quad+\left(d \delta_{2}-r \delta_{1}\right) \int_{0}^{L} u_{x} \widehat{\theta} d x+\left(d \delta_{1}-c \delta_{2}\right) \int_{0}^{L} u_{x} \widehat{P} d x \\
& \quad+\int_{0}^{L}\left(\delta_{1} \theta+\delta_{2} P\right) \widehat{u}_{x} d x, \\
& \Gamma(\widehat{w}, \widehat{u}, \widehat{\theta}, \widehat{P})=\int_{0}^{L} h_{1} \widehat{w} d x+\int_{0}^{L} h_{2} \widehat{u} d x+\int_{0}^{L} h_{3} \widehat{\theta} d x+\int_{0}^{L} h_{4} \widehat{P} d x .
\end{aligned}
$$


Now, for $\mathscr{H}_{1}=H_{0}^{2}(0, L) \times H_{0}^{1}(0, L) \times L^{2}(0, L) \times L^{2}(0, L)$, equipped with the norm

$$
\begin{aligned}
\|(w, u, \theta, P)\|_{\mathscr{C}_{1}}^{2}= & \|w\|_{2}^{2}+\left\|w_{x x}\right\|_{2}^{2}+\|u\|_{2}^{2}+\left\|u_{x}\right\|_{2}^{2}+\|\theta\|_{2}^{2} \\
& +\left\|\theta_{x}\right\|_{2}^{2}+\left\|P_{x}\right\|_{2}^{2}+\|P\|_{2}^{2},
\end{aligned}
$$

then, we have

$$
\begin{aligned}
B((w, u, \theta, P),(w, u, \theta, P)) & \\
= & \mu_{3} \int_{0}^{L} w^{2} d x+d_{2} \int_{0}^{L} w_{x x}^{2} d x+\int_{0}^{L} u^{2} d x+d_{1} \int_{0}^{L} u_{x}^{2} d x \\
& +\alpha_{1} \int_{0}^{L} \theta^{2} d x+\alpha_{2} \int_{0}^{L} P^{2} d x+r C_{\beta} \int_{0}^{L} \theta_{x}^{2} d x+c C_{\lambda} \int_{0}^{L} P_{x}^{2} d x \\
& -\left(d C_{\beta}+d C_{\lambda}\right) \int_{0}^{L} P_{x} \theta_{x} d x+\left(d \delta_{2}-r \delta_{1}\right) \int_{0}^{L} u_{x} \theta d x \\
& +\left(d \delta_{1}-c \delta_{2}\right) \int_{0}^{L} u_{x} P d x+\int_{0}^{L}\left(\delta_{1} \theta+\delta_{2} P\right) u_{x} d x .
\end{aligned}
$$

Then, for some $M_{0}>0$,

$$
B((w, u, \theta, P),(w, u, \theta, P)) \geq M_{0}\|(w, u, \theta, P)\|_{\mathscr{H}_{1}}^{2} .
$$

Thus, $B$ is coercive. obtain

By Cauchy-Schwarz's and Poincaré's inequalities, we

$$
\begin{aligned}
& B((w, u, \theta, P),(\widehat{w}, \widehat{u}, \widehat{\theta}, \widehat{P})) \\
& \quad \leq M_{1}\|(w, u, \theta, P)\|_{\mathscr{H}_{1}}^{2}\|(\widehat{w}, \widehat{u}, \widehat{\theta}, \widehat{P})\|_{\mathscr{H}_{1}}^{2} .
\end{aligned}
$$

Similarly, we get

$$
\Gamma(\widehat{w}, \widehat{u}, \widehat{\theta}, \widehat{P}) \leq M_{2}\|(\widehat{w}, \widehat{u}, \widehat{\theta}, \widehat{P})\|_{\mathscr{H}_{1}}^{2} .
$$

Consequently, applying the Lax-Milgram theorem, problem (84) admits a unique solution $(w, u, \theta, P) \in \mathscr{H}_{1}$, for all $(\widehat{w}, \widehat{u}, \widehat{\theta}, \widehat{P}) \in \mathscr{H}_{1}$. Applying the classical elliptic regularity, it follows from (80) that $(w, u, \theta, P) \in \mathscr{H}_{1}$.

Therefore, the operator $I-\mathscr{A}(t)$ is surjective for any fixed $t>0$. Since $\kappa(t)>0$ and

$$
I-\overline{\mathscr{A}}(t)=(1+\kappa(t)) I-\mathscr{A}(t),
$$

we deduce that the operator $I-\bar{A}(t)$ is also surjective for any $t>0$.

To complete the proof of (3), it suffices to show that

$$
\frac{\|U\|_{t}}{\|U\|_{s}} \leq e^{\left(c / 2 \tau_{0}\right)|t-s|}, \quad \forall t, s \in[0, T]
$$

where $U=(w, \varphi, u, \psi, \theta, \eta, P, v, z)^{T}$ and $\|.\|_{t}$ is the norm associated with the inner product (56).
For $t, s \in[0, T]$, we have from (56) that

$$
\begin{aligned}
\|U\|_{t}^{2}- & \|U\|_{s}^{2} e^{\left(c / \tau_{0}\right)|t-s|} \\
= & \left(1-e^{\left(c / \tau_{0}\right)|t-s|}\right) \int_{0}^{L}\left\{\varphi^{2}+d_{2} w_{x x}^{2}+d_{1} u_{x}^{2}+\psi^{2}\right\} d x \\
& +\left(1-e^{\left(c / \tau_{0}\right)|t-s|}\right)<\Lambda(\theta, P)^{T},(\theta, P)^{T}> \\
& +\left(1-e^{\left(c / \tau_{0}\right)|t-s|}\right)\left\{\|\eta\|_{\mathscr{M}_{\beta}}^{2}+\|v\|_{\mathscr{M}_{\lambda}}^{2}\right\} \\
& +\xi\left(\tau(t)-\tau(s) e^{\left(c / \tau_{0}\right)|t-s|}\right) \int_{0}^{L} \int_{0}^{1} z^{2}(x, \rho, t) d \rho d x .
\end{aligned}
$$

It is clear that $\left(1-e^{\left(c / \tau_{0}\right)|t-s|}\right) \leq 0$. Now, we will prove that $\left(\tau(t)-\tau(s) e^{\left(c / \tau_{0}\right)|t-s|}\right) \leq 0$ for $c>0$. To do this, we have

$$
\tau(t)=\tau(s)+\tau^{\prime}(a)(t-s),
$$

where $a \in(s, t)$, which implies

$$
\frac{\tau(t)}{\tau(s)} \leq 1+\frac{|\tau(a)|}{\tau(s)}|t-s| .
$$

By using (11), we deduce that

$$
\frac{\tau(t)}{\tau(s)} \leq 1+\frac{c}{\tau_{0}}|t-s| \leq e^{\left(c / \tau_{0}\right)|t-s|},
$$

which proves (92); therefore, this completes the proof of (3).

(4) It is clear that

$$
\frac{d}{d t} \mathscr{A}(t) U=\left(\begin{array}{c}
0 \\
0 \\
0 \\
0 \\
0 \\
0 \\
0 \\
0 \\
\frac{\left(\tau^{\prime \prime}(t) \tau^{\prime}(t) \rho-\tau^{\prime}(t)\left(\tau^{\prime}(t) \rho-1\right)\right)}{\tau^{2}(t)} z_{\rho}
\end{array}\right) .
$$

Then, by (11) and (25), (4) holds exactly as in [1]. Consequently, from the above analysis, we deduce that the problem

$$
\left\{\begin{array}{l}
\bar{U}_{t}=\overline{\mathscr{A}}(t) \bar{U}_{t}, \\
\bar{U}_{t}(0)=U_{0},
\end{array}\right.
$$


has a solution $\bar{U} \in C([0, \infty), \mathscr{H})$, and if $U_{0} \in \mathscr{D}(\mathscr{A}(0))$, then

$$
\bar{U} \in C([0, \infty), \mathscr{D}(\mathscr{A}(0))) \cap C^{1}([0, \infty), \mathscr{H}) .
$$

Now, let

$$
U(t)=e^{9(t)} \bar{U}(t),
$$

with $\vartheta(t)=\int_{0}^{t} \kappa(s) d s$; then, by using (98), we have

$$
\begin{aligned}
U_{t}(t) & =\kappa(t) e^{9(t)} \bar{U}(t)+e^{\vartheta(t)} \bar{U}_{t}(t) \\
& =\kappa(t) e^{\vartheta(t)} \bar{U}(t)+e^{\vartheta(t)} \overline{\mathscr{A}}(t) \bar{U}(t) \\
& =e^{\vartheta(t)}(\kappa(t) \bar{U}(t)+\overline{\mathscr{A}}(t) \bar{U}(t)) \\
& \left.=e^{9(t)}(\mathscr{A}(t) \bar{U}(t))=\mathscr{A}(t) U(t)\right) .
\end{aligned}
$$

Consequently, $U(t)$ is the unique solution of (46).

It remains to prove that the operator $\mathscr{F}$ defined in (48) is locally Lipschitz in $\mathscr{H}$.

Let $U_{1}=\left(w_{1}, \varphi_{1}, u_{1}, \psi_{1}, \theta_{1}, \eta_{1}, P_{1}, v_{1}, z_{1}\right)^{T} \in \mathscr{H}$ and $U_{2}=$ $\left(w_{2}, \varphi_{2}, u_{2}, \psi_{2}, \theta_{2}, \eta_{2}, P_{2}, v_{2}, z_{2}\right)^{T} \in \mathscr{H}$. Then, we have

$$
\left\|\mathscr{F}\left(U_{1}\right)-\mathscr{F}\left(U_{2}\right)\right\|=d_{1}\left(|R|^{2}+|K|^{2}\right),
$$

where

$$
\begin{aligned}
R & =\left[\left(u_{1 x}+\frac{1}{2} w_{1 x}^{2}\right) w_{1 x}-\left(u_{2 x}+\frac{1}{2} w_{2 x}^{2}\right) w_{2 x}\right], \\
K & =\frac{1}{2}\left(w_{1 x}^{2}-w_{2 x}^{2}\right) .
\end{aligned}
$$

Adding and subtracting the term $\left(u_{1 x}+(1 / 2) w_{1 x}^{2}\right) w_{2 x}$ inside the norm $|R|$, we find

$$
\begin{aligned}
|R| \leq & \left\|w_{1 x}-w_{2 x}\right\|_{L^{\infty}(0, L)}\left|u_{1 x}+\frac{1}{2} w_{1 x}^{2}\right|+\left\|w_{2 x}\right\|_{L^{\infty}}\left|u_{1 x}-u_{2 x}\right| \\
& +\frac{1}{2}\left\|w_{2 x}\right\|_{L^{\infty}}\left|w_{1 x}+w_{2 x}\right|\left\|w_{1 x}-w_{2 x}\right\|_{L^{\infty}(0, L)} .
\end{aligned}
$$

Using the embedding of $H^{1}(0, L)$ into $L^{\infty}(0, L)$, from (104), one has

$$
|R| \leq k_{1}\left(\left\|U_{1}\right\|_{\mathscr{H}},\left\|U_{2}\right\|_{\mathscr{H}}\right)\left\|U_{1}-U_{2}\right\| .
$$

Using once again the embedding of $H^{1}(0, L)$ into $L^{\infty}(0, L)$, one also sees that

$$
|K| \leq k_{2}\left(\left\|U_{1}\right\|_{\mathscr{H}},\left\|U_{2}\right\|_{\mathscr{H}}\right)\left\|U_{1}-U_{2}\right\| .
$$

Combining (102), (105), and (106), consequently, $\mathscr{F}(U)$ is locally Lipschitz continuous in $\mathscr{H}$. This ends the proof of Theorem 1.

\section{General Decay}

In this section, we state and prove the stability of system (21)-(23) using the multiplier technique under the assumptions (26)-(31).

We define the energy functional $E$ by

$$
\begin{aligned}
E(t)= & \frac{1}{2} \int_{0}^{L}\left\{w_{t}^{2}+u_{t}^{2}+d_{2} w_{x x}^{2}+d_{1}\left(u_{x}+\frac{1}{2} w_{x}^{2}\right)^{2}+c \theta^{2}+r P^{2}\right\} d x \\
& +d<\theta, P>+\frac{1}{2}\|\eta\|_{\mathscr{M}_{\beta}}^{2}+\frac{1}{2}\|v\|_{\mathscr{M}_{\lambda}}^{2} \\
& +\frac{\xi}{2} \int_{0}^{L} \int_{0}^{1} \tau(t) z^{2}(x, \rho, t) d \rho d x,
\end{aligned}
$$

where

$$
\frac{\left|\mu_{2}\right|}{\sqrt{1-d}} \leq \xi \leq\left(2 \mu_{1}-\frac{\left|\mu_{2}\right|}{\sqrt{1-d}}\right)
$$

The following lemma shows that the energy is decreasing.

Lemma 3. Assume that (26)-(31) hold and the hypotheses (7), (11), and (25) are satisfied. Then, for $\forall C \geq 0$,

$$
\begin{aligned}
E^{\prime}(t) \leq & -C\left(\int_{0}^{L} w_{t}^{2} d x+\int_{0}^{L} z^{2}(x, 1, t) d x\right)-\frac{\alpha_{1}}{4}\|\eta\|_{\mathscr{M}_{\beta}}^{2} \\
& +\frac{1}{4} \int_{0}^{\infty} \beta^{\prime}(\sigma)\left\|\eta_{x}(\sigma)\right\|^{2} d \sigma-\frac{\alpha_{2}}{4}\|v\|_{\mathscr{M}_{\lambda}}^{2} \\
& +\frac{1}{4} \int_{0}^{\infty} \lambda^{\prime}(\sigma)\left\|v_{x}(\sigma)\right\|^{2} d \sigma \leq 0 .
\end{aligned}
$$

Proof. Multiplying the equations of (21) by $w_{t}, u_{t}, \theta, \eta, P, v$, and $\xi z$, respectively, then by integration by parts, we get

$$
\begin{aligned}
\frac{1}{2} \frac{d}{d t} \int_{0}^{L}\left\{w_{t}^{2}+u_{t}^{2}+d_{2} w_{x x}^{2}+d_{1}\left(u_{x}+\frac{1}{2} w_{x}^{2}\right)^{2}+c \theta^{2}+r P^{2}\right\} d x \\
\quad+\frac{d}{d t}\left\{d<\theta, P>+\frac{1}{2}\|\eta\|_{\mathscr{M}_{\beta}}^{2}+\frac{1}{2}\|v\|_{\mathscr{M}_{\lambda}}^{2}\right\} \\
\quad+\frac{\xi}{2} \frac{d}{d t} \int_{0}^{L} \int_{0}^{1} \tau(t) z^{2}(x, \rho, t) d \rho d x \\
=-\mu_{1} \int_{0}^{1} \omega_{t}^{2} d x-\mu_{2} \int_{0}^{L} w_{t} z(x, 1, t) d x \\
\quad+\frac{1}{2} \int_{0}^{\infty} \beta^{\prime}(\sigma)\left\|\eta_{x}(\sigma)\right\|^{2} d \sigma+\frac{1}{2} \int_{0}^{\infty} \lambda^{\prime}(\sigma)\left\|v_{x}(\sigma)\right\|^{2} d \sigma \\
\quad+\frac{\xi}{2} \int_{0}^{L} \int_{0}^{1} \tau^{\prime}(t) z^{2}(x, \rho, t) d \rho d x \\
\quad-\xi \int_{0}^{L} \int_{0}^{1}\left(1-\tau^{\prime}(t) \rho\right) z(x, \rho, t) z_{\rho}(x, \rho, t) d \rho d x \\
\leq-\mu_{1} \int_{0}^{1} \omega_{t}^{2} d x-\mu_{2} \int_{0}^{L} w_{t} z(x, 1, t) d x-\frac{\alpha_{1}}{4}\|\eta\|_{\mathscr{M}_{\beta}}^{2}-\frac{\alpha_{2}}{4}\|v\|_{\mathscr{M}_{\lambda}}^{2}
\end{aligned}
$$




$$
\begin{aligned}
& +\frac{1}{4} \int_{0}^{\infty} \beta^{\prime}(\sigma)\left\|\eta_{x}(\sigma)\right\|^{2} d \sigma+\frac{1}{4} \int_{0}^{\infty} \lambda^{\prime}(\sigma)\left\|v_{x}(\sigma)\right\|^{2} d \sigma \\
& -\frac{\xi}{2} \int_{0}^{L} \int_{0}^{1} \frac{d}{d \rho}\left(\left(1-\tau^{\prime}(t) \rho\right) z^{2}(x, \rho, t)\right) d \rho d x \\
& =-\mu_{1} \int_{0}^{1} \omega_{t}^{2} d x-\mu_{2} \int_{0}^{L} w_{t} z(x, 1, t) d x-\frac{\alpha_{1}}{4}\|\eta\|_{\mathscr{M}_{\beta}}^{2}-\frac{\alpha_{2}}{4}\|v\|_{\mathscr{M}_{\lambda}}^{2} \\
& +\frac{1}{4} \int_{0}^{\infty} \beta^{\prime}(\sigma)\left\|\eta_{x}(\sigma)\right\|^{2} d \sigma+\frac{1}{4} \int_{0}^{\infty} \lambda^{\prime}(\sigma)\left\|v_{x}(\sigma)\right\|^{2} d \sigma \\
& +\frac{\xi}{2} \int_{0}^{L}\left(z^{2}(x, 0, t)-z^{2}(x, 1, t)\right) d x \\
& \left.+\frac{\xi \tau^{\prime}(t)}{2} \int_{0}^{L} z^{2}(x, 1, t)\right) d x .
\end{aligned}
$$

From (110), we find

$$
\begin{aligned}
E^{\prime}(t) \leq & -\left(\mu_{1}-\frac{\xi}{2}\right) \int_{0}^{L} w_{t}^{2} d x+\left(\frac{\xi \tau^{\prime}(t)}{2}-\frac{\xi}{2}\right) \int_{0}^{L} z^{2}(x, 1, t) d x \\
& -\mu_{2} \int_{0}^{L} w_{t} z(x, 1, t) d x-\frac{\alpha_{1}}{4}\|\eta\|_{\mathscr{M}_{\beta}}^{2}-\frac{\alpha_{2}}{4}\|v\|_{\mathscr{M}_{\lambda}}^{2} \\
& +\frac{1}{4} \int_{0}^{\infty} \beta^{\prime}(\sigma)\left\|\eta_{x}(\sigma)\right\|^{2} d \sigma+\frac{1}{4} \int_{0}^{\infty} \lambda^{\prime}(\sigma)\left\|v_{x}(\sigma)\right\|^{2} d \sigma .
\end{aligned}
$$

Using Young's inequality, we have

$$
\begin{aligned}
-\mu_{2} \int_{0}^{L} w_{t} z(x, 1, t) d x \leq & \frac{\left|\mu_{2}\right|}{2 \sqrt{1-d}} \int_{0}^{L} w_{t}^{2} d x \\
& +\frac{\left|\mu_{2}\right| \sqrt{1-d}}{2} \int_{0}^{L} z^{2}(x, 1, t) d x .
\end{aligned}
$$

Inserting (112) into (111), we get

$$
\begin{aligned}
E^{\prime}(t) \leq & -\left(\mu_{1}-\frac{\xi}{2}-\frac{\left|\mu_{2}\right|}{2 \sqrt{1-d}}\right) \int_{0}^{L} w_{t}^{2} d x \\
& +\left(\frac{\xi}{2}\left(\tau^{\prime}(t)-1\right)+\frac{\left|\mu_{2}\right| \sqrt{1-d}}{2}\right) \int_{0}^{L} z^{2}(x, 1, t) d x \\
& -\frac{\alpha_{1}}{4}\|\eta\|_{\mathscr{M}_{\beta}}^{2}+\frac{1}{4} \int_{0}^{\infty} \beta^{\prime}(\sigma)\left\|\eta_{x}(\sigma)\right\|^{2} d \sigma-\frac{\alpha_{2}}{4}\|v\|_{\mathscr{M}_{\lambda}}^{2} \\
& +\frac{1}{4} \int_{0}^{\infty} \lambda^{\prime}(\sigma)\left\|v_{x}(\sigma)\right\|^{2} d \sigma .
\end{aligned}
$$

Then, by using (7), (28)-(31), and (108), we obtain (109).

In the following, we state and prove our stability result; we introduce and prove several lemmas.
Lemma 4. The functional

$$
F_{1}(t):=\int_{0}^{L}\left(u_{t} u+\frac{1}{2} w_{t} w+\frac{\beta_{1}}{4} w^{2}\right) d x,
$$

satisfies, for any $\varepsilon_{1}>0$,

$$
\begin{aligned}
F_{1}^{\prime}(t) \leq & -d_{1} \int_{0}^{L}\left(u_{x}+\frac{1}{2} w_{x}^{2}\right)^{2} d x-\frac{d_{2}}{4} \int_{0}^{L} w_{x x}^{2} d x+\int_{0}^{L} u_{t}^{2} d x \\
& +\frac{1}{2} \int_{0}^{L} w_{t}^{2} d x+2 \varepsilon_{1} \int_{0}^{L} u_{x}^{2} d x+\frac{\delta_{1}^{2}}{4 \varepsilon_{1}} \int_{0}^{L} \theta^{2}+\frac{\delta_{2}^{2}}{4 \varepsilon_{1}} \int_{0}^{L} P^{2} \\
& +c \int_{0}^{L} z^{2}(x, 1, t) d x .
\end{aligned}
$$

Proof. By differentiating $F_{1}$, then by integration by parts, we obtain

$$
\begin{aligned}
F_{1}^{\prime}(t)= & \int_{0}^{L} u_{t}^{2} d x+\frac{1}{2} \int_{0}^{L} w_{t}^{2} d x-\frac{1}{2} d_{1} \int_{0}^{L}\left(u_{x}+\frac{1}{2} w_{x}^{2}\right) w_{x}^{2} d x \\
& -d_{1} \int_{0}^{L} u_{x}\left(u_{x}+\frac{1}{2} w_{x}^{2}\right) d x-\frac{\mu_{2}}{2} \int_{0}^{L} w z(x, 1, t) d x \\
& -\frac{d_{2}}{2} \int_{0}^{L} w_{x x}^{2} d x+\delta_{1} \int_{0}^{L} \theta u_{x} d x+\delta_{2} \int_{0}^{L} P u_{x} d x .
\end{aligned}
$$

In what follows, using Young's and Poincaré's inequalities, we obtain (115).

Then, we have the following lemma.

Lemma 5. The functional

$$
F_{2}(t):=\int_{0}^{L} u_{t} \Phi d x
$$

where $-\delta_{1} \Phi_{x}=c \theta+d P$, with $\Phi(0)=\Phi(L)=0$, satisfies

$$
\begin{aligned}
F_{2}^{\prime}(t) \leq & -\int_{0}^{L} u_{t}^{2} d x+\varepsilon_{2} \int_{0}^{L}\left(u_{x}+\frac{1}{2} w_{x}^{2}\right)^{2} d x+c\|\eta\|_{\mathscr{\mu}}^{2} \\
& +c\left(1+\frac{1}{\varepsilon_{2}}\right) \int_{0}^{L} \theta^{2} d x+c\left(1+\frac{1}{\varepsilon_{2}}\right) \int_{0}^{L} P^{2} d x
\end{aligned}
$$

Proof. For direct computations, we have

$$
\begin{gathered}
F_{2}^{\prime}(t)=\underbrace{\int_{0}^{L} u_{t t} \Phi d x}_{f} \\
1(t)+\int_{0}^{L} u_{t} \Phi_{t} d x .
\end{gathered}
$$


Using Young's inequality and integrating by parts, we obtain

$$
\begin{aligned}
f_{1}(t) \leq & \varepsilon_{2} \int_{0}^{L}\left(u_{x}+\frac{1}{2} w_{x}^{2}\right)^{2} d x+c\left(1+\frac{1}{\varepsilon_{2}}\right) \int_{0}^{L} \theta^{2} d x \\
& +c\left(1+\frac{1}{\varepsilon_{2}}\right) \int_{0}^{L} P^{2} d x, \\
f_{2}(t)=- & \frac{1}{\delta_{1}} \int_{0}^{L} u_{t} \partial_{x}^{-1}\left(\int_{0}^{\infty} \beta(\sigma) \eta_{x x}(\sigma) d \sigma+\delta_{1} u_{t x}\right) d x \\
= & -\frac{1}{\delta_{1}} \int_{0}^{L} u_{t}\left(\int_{0}^{\infty} \beta(\sigma) \eta_{x}(\sigma) d \sigma+\delta_{1} u_{t}\right) d x \\
\leq & -\int_{0}^{L} u_{t}^{2} d x+c\|\eta\|_{\mathscr{M}_{\beta} .}^{2}
\end{aligned}
$$

From (120) and (121), we obtain (118).

Lemma 6. Assuming that assumptions (31) and (32) hold, the functional

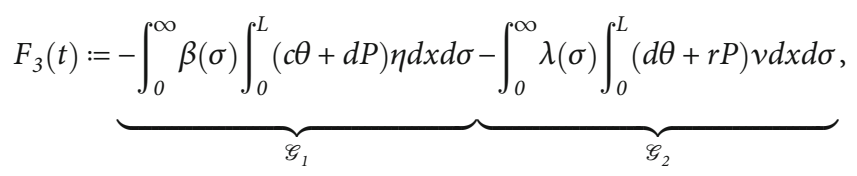

satisfies

$$
\begin{aligned}
F_{3}^{\prime}(t) \leq & -\widehat{c} \int_{0}^{L} \theta^{2} d x-\widehat{r} \int_{0}^{L} P^{2} d x+\beta_{0}\|\eta\|_{\mathscr{M}_{\beta}}^{2}+\lambda_{0}\|v\|_{\mathscr{M}_{\lambda}}^{2} \\
& +\frac{c}{\varepsilon_{3}} \int_{0}^{L} u_{t}^{2} d x-C_{\beta_{0}} \int_{0}^{\infty} \beta^{\prime}(\sigma)\left\|\eta_{x}(\sigma)\right\|^{2}(\sigma) d \sigma \\
& -C_{\lambda_{0}} \int_{0}^{\infty} \lambda^{\prime}(\sigma)\left\|v_{x}(\sigma)\right\|^{2}(\sigma) d \sigma
\end{aligned}
$$

where

$$
\begin{aligned}
& \widehat{c}=\frac{1}{2}\left(\beta_{0} c-\left(\beta_{0}+\lambda_{0}\right) \frac{d}{\zeta}\right), \\
& \widehat{r}=\frac{1}{2}\left(\lambda_{0} r-\left(\mu_{0}+\lambda_{0}\right) d \zeta\right),
\end{aligned}
$$

and $\zeta>0$ satisfies (29).

Proof. We take the derivative of $F_{3}=\mathscr{G}_{1}+\mathscr{G}_{2}$, which gives

$$
\begin{aligned}
\mathscr{G}_{1}^{\prime}(t)= & -\int_{0}^{\infty} \beta(\sigma) \int_{0}^{L}(c \theta+d P)_{t} \eta d x d \sigma \\
& -\int_{0}^{\infty} \beta(\sigma) \int_{0}^{L}(c \theta+d P) \eta_{t} d x d \sigma \\
= & -\int_{0}^{\infty} \beta(\sigma) \int_{0}^{L}\left(c \theta_{t}+d P_{t}\right) \eta d x d \sigma+c \int_{0}^{\infty} \beta(\sigma) \int_{0}^{L} \theta \eta_{\sigma} d x d \sigma \\
& +d \int_{0}^{\infty} \beta(\sigma) \int_{0}^{L} P \eta_{\sigma} d x d \sigma-c \beta_{0} \int_{0}^{L} \theta^{2} d x-d \int_{0}^{\infty} \beta(\sigma) \int_{0}^{L} P \theta d x d \sigma .
\end{aligned}
$$

The first term on the right-hand side of (125) is

$$
\begin{aligned}
-\int_{0}^{\infty} \beta(\sigma) \int_{0}^{L}(c \theta+d P)_{t} \eta d x d \sigma \\
=-\delta_{1} \int_{0}^{\infty} \beta(\sigma) \int_{0}^{L} u_{t x} \eta d x d \sigma \\
\quad-\int_{0}^{L}\left(\int_{0}^{\infty} \beta(\sigma) \eta_{x x} d \sigma\right)\left(\int_{0}^{\infty} \beta(\sigma) \eta d \sigma\right) d x
\end{aligned}
$$

and can be controlled in the following way:

$$
\left|-\delta_{1} \int_{0}^{\infty} \beta(\sigma) \int_{0}^{L} u_{t x} \eta d x d \sigma\right| \leq C\left(\varepsilon_{3}\right)\|\eta\|_{\mathscr{M}_{\beta}}^{2}+\frac{c}{\varepsilon_{3}} \int_{0}^{L} u_{t}^{2} d x
$$

$$
-\int_{0}^{L}\left(\int_{0}^{\infty} \beta(\sigma) \eta_{x x} d \sigma\right)\left(\int_{0}^{\infty} \beta(\sigma) \eta d \sigma\right) d x \leq \beta_{0}\|\eta\|_{\mu_{\beta}}^{2} .
$$

Moreover, by integration by parts, we get

$$
\begin{aligned}
\left|c \int_{0}^{\infty} \beta(\sigma) \int_{0}^{L} \theta \eta_{\sigma} d x d \sigma\right| & =c\left|-\int_{0}^{\infty} \beta^{\prime}(\sigma) \int_{0}^{L} \theta \eta d x d \sigma\right| \\
& \leq \frac{c \mu_{0}}{4} \int_{0}^{L} \theta^{2} d x-C_{\beta_{0}} \int_{0}^{\infty} \beta^{\prime}(\sigma)\left\|\eta_{x}(\sigma)\right\|^{2} d \sigma,
\end{aligned}
$$

where $C_{\beta_{0}}>0$. Similarly, we obtain

$$
\begin{aligned}
\left|d \int_{0}^{\infty} \beta(\sigma) \int_{0}^{L} P \eta_{\sigma} d x d \sigma\right| & =c\left|-\int_{0}^{\infty} \beta^{\prime}(\sigma) \int_{0}^{L} P \eta d x d \sigma\right| \\
& \leq \frac{r \lambda_{0}}{4} \int_{0}^{L} P^{2} d x-C_{\beta_{0}}^{\prime} \int_{0}^{\infty} \beta^{\prime}(\sigma)\left\|\eta_{x}(\sigma)\right\|^{2} d \sigma
\end{aligned}
$$

where $C_{\beta_{0}}^{\prime}>0$. Using (29), we get

$$
-d \int_{0}^{\infty} \beta(\sigma)\left(\int_{0}^{L} \theta P d x\right) d \sigma \leq \beta_{0} \frac{d}{2 \zeta} \int_{0}^{L} \theta^{2} d x+\beta_{0} \frac{d \zeta}{2} \int_{0}^{L} P^{2} d x
$$

Then, we obtain

$$
\begin{aligned}
\mathscr{G}_{1}^{\prime}(t) \leq & \frac{\beta_{0}}{2}\left(\frac{d}{\zeta}-\frac{3 c}{2}\right) \int_{0}^{L} \theta^{2} d x+\frac{1}{2}\left(\beta_{0} d \zeta+\frac{r \lambda_{0}}{2}\right) \int_{0}^{L} P^{2} d x \\
& +\frac{c}{\varepsilon_{3}} \int_{0}^{L} u_{t}^{2} d x-\mathscr{C}_{\beta_{0}} \int_{0}^{\infty} \beta^{\prime}(\sigma)\left\|\eta_{x}(\sigma)\right\|^{2} d \sigma \\
& +\left(\beta_{0}+C\left(\varepsilon_{3}\right)\|\eta\|_{\mathscr{M}_{\beta}}^{2},\right.
\end{aligned}
$$


where $\mathscr{C}_{\beta_{0}}=C_{\beta_{0}}+C_{\beta_{0}}^{\prime}$. Then, using the same arguments, we find

$$
\begin{aligned}
\mathscr{G}_{2}^{\prime}(t) \leq & \frac{1}{2}\left(\lambda_{0} \frac{d}{\zeta}+\frac{\beta_{0} c}{2}\right) \int_{0}^{L} \theta^{2} d x+\frac{\lambda_{0}}{2}\left(d \zeta-\frac{3 r}{2}\right) \int_{0}^{L} P^{2} d x \\
& +\frac{c}{\varepsilon_{3}} \int_{0}^{L} u_{t}^{2} d x-\mathscr{C}_{\lambda_{0}} \int_{0}^{\infty} \lambda^{\prime}(\sigma)\left\|\nu_{x}(\sigma)\right\|^{2} d \sigma \\
& +\left(\lambda_{0}+C\left(\varepsilon_{3}\right)\|v\|_{\mathscr{M}_{\lambda}}^{2} .\right.
\end{aligned}
$$

Adding (127) and (133), we obtain (123).

We choose $\zeta$ in such a way that

$$
\begin{aligned}
& \widehat{c}=\frac{1}{2}\left(\beta_{0} c-\left(\beta_{0}+\lambda_{0}\right) \frac{d}{\zeta}\right)>0, \\
& \widehat{r}=\frac{1}{2}\left(\lambda_{0} r-\left(\beta_{0}+\lambda_{0}\right) d \zeta\right)>0,
\end{aligned}
$$

which implies

$$
\frac{d}{c}<\frac{\beta_{0}+\lambda_{0}}{\beta_{0}} \frac{d}{c}<\zeta<\frac{\lambda_{0}}{\beta_{0}+\lambda_{0}} \frac{r}{d}<\frac{r}{d}
$$

Then, $\zeta$ satisfies (29).

Now, let us introduce the following functional.

Lemma 7. The functional

$$
F_{4}(t):=\xi \tau(t) \int_{0}^{L} \int_{0}^{1} e^{-2 \tau(t) \rho} z^{2}(x, \rho, t) d \rho d x,
$$

satisfies

$$
F_{4}^{\prime}(t) \leq-2 F_{4}(t)-\eta_{1} \int_{0}^{L} z^{2}(x, 1, t) d x+\xi \int_{0}^{L} w_{t}^{2} d x
$$

where $\eta_{1}$ is a positive constant.

Proof. By differentiating $F_{4}$, with respect to $t$, we have

$$
\begin{aligned}
F_{4}^{\prime}(t)= & \xi \tau^{\prime}(t) \int_{0}^{L} \int_{0}^{1} e^{-2 \tau(t) \rho} z^{2}(x, \rho, t) d \rho d x \\
& +\xi \tau(t) \int_{0}^{L} \int_{0}^{1}\left\{-2 \tau^{\prime}(t) \rho e^{-2 \tau(t) \rho} z^{2}+e^{-2 \tau(t) \rho} z_{t} z\right\} d \rho d x .
\end{aligned}
$$

By using the last equation of (21), we have

$$
\begin{aligned}
& \tau(t) \int_{0}^{L} \int_{0}^{1} e^{-2 \tau(t) \rho} z_{t} z d \rho d x \\
& =\int_{0}^{L} \int_{0}^{1}\left(\tau^{\prime}(t) \rho-1\right) e^{-2 \tau(t) \rho} z_{\rho} z d \rho d x \\
& =\frac{1}{2} \int_{0}^{L} \int_{0}^{1} \frac{d}{d \rho}\left\{\left(\tau^{\prime}(t) \rho-1\right) e^{-2 \tau(t) \rho} z^{2}\right\} d \rho d x \\
& \quad+\tau(t) \int_{0}^{L} \int_{0}^{1}\left(\tau^{\prime}(t) \rho-1\right) e^{-2 \tau(t) \rho} z^{2} d \rho d x \\
& \quad-\frac{\tau^{\prime}(t)}{2} \int_{0}^{L} \int_{0}^{1} e^{-2 \tau(t) \rho} z^{2} d x .
\end{aligned}
$$

Using (137)-(139), we get

$$
\begin{aligned}
F_{4}^{\prime}(t)= & -2 \xi \tau(t) \int_{0}^{L} \int_{0}^{1} e^{-2 \tau(t) \rho} z^{2}(x, \rho, t) d \rho d x+\xi \int_{0}^{L} z^{2}(x, 0, t) d x \\
& -\xi\left(1-\tau^{\prime}(t)\right) e^{-2 \tau(t)} \int_{0}^{L} z^{2}(x, 1, t) d x .
\end{aligned}
$$

Then, by using (7), (25), and the fact that $z(x, 0, t)=$ $w_{t}(x, t)$ and setting $\eta_{1}=\xi(1-d) e^{-2 \bar{\tau}}$, we obtain (137).

We are now ready to prove the following result.

Theorem 8. Assume (26)-(31) hold; there exist positive constants $C_{1}$ and $C_{2}$ such that the energy functional given by (107) satisfies

$$
E(t) \leq C_{2} e^{-C_{1} t}, \quad \forall t \geq 0 .
$$

Proof. We define a Lyapunov functional

$$
\mathscr{L}(t):=N E(t)+\sum_{i=1}^{i=3} N_{i} F_{i}(t)+F_{4}(t)
$$

where $N$ and $N_{i}, i=1,2,3$, are positive constants to be selected later.

By differentiating (142) and using (109), (115), (118), (123), and (137), including the relation

$$
\begin{aligned}
\int_{0}^{L} u_{x}^{2} d x & =\int_{0}^{L}\left(u_{x}^{2}+\frac{1}{2} w_{x}^{2}-\frac{1}{2} w_{x}^{2}\right) d x \\
& \leq 2 \int_{0}^{L}\left(u_{x}+\frac{1}{2} w_{x}^{2}\right)^{2} d x-\frac{1}{2} \int_{0}^{L} w_{x}^{4} d x \\
& \leq 2 \int_{0}^{L}\left(u_{x}+\frac{1}{2} w_{x}^{2}\right)^{2} d x-\frac{L}{4} \int_{0}^{L} w_{x x}^{2} d x,
\end{aligned}
$$


we get

$$
\begin{aligned}
\mathscr{L}^{\prime}(t) \leq & -\left[\left(d_{1}-2 \varepsilon_{1}\right) N_{1}-\varepsilon_{2} N_{2}\right] \int_{0}^{L}\left(u_{x}+\frac{1}{2} w_{x}^{2}\right)^{2} d x \\
& -\left[N_{2}-N_{1}-\frac{c}{\varepsilon_{3}} N_{3}\right] \int_{0}^{L} u_{t}^{2} d x \\
& -\left[\left(\frac{d_{2}}{4}-\frac{L}{2} \varepsilon_{1}\right) N_{1}\right] \int_{0}^{L} w_{x x}^{2} d x \\
& -\left[C N-\frac{1}{2} N_{1}-\xi\right] \int_{0}^{L} \omega_{t}^{2} d x \\
& -\left[\widehat{c} N_{3}-\frac{\delta_{1}^{2}}{4 \varepsilon_{1}} N_{1}-c\left(1+\frac{1}{\varepsilon_{2}}\right) N_{2}\right] \int_{0}^{L} \theta^{2} d x \\
& -\left[\widehat{r} N_{3}-\frac{\delta_{2}^{2}}{4 \varepsilon_{1}} N_{1}-c\left(1+\frac{1}{\varepsilon_{2}}\right) N_{2}\right] \int_{0}^{L} P^{2} d x \\
& -\left[C N-c N_{1}+\eta_{1}\right] \int_{0}^{L} z^{2}(x, 1, t) d x-2 F_{4}(t) \\
& -\left[\frac{\alpha_{1}}{4} N-c N_{2}-\mu_{0} N_{3}\right]\|\eta\|_{\mathscr{M}_{\beta}}^{2} \\
& \left.-\left[\frac{\alpha_{2}}{4} N-\lambda_{0} N_{3}\right]\|v\|_{\mathscr{M}_{\lambda}}^{2} N-C_{\lambda_{0}} N_{3}\right] \int_{0}^{\infty} \lambda^{\prime}(\sigma)\left\|v_{x}(\sigma)\right\|^{2} d \sigma . \\
& {\left[\frac{1}{4} N-C_{\mu_{0}} N_{3}\right] \int_{0}^{\infty} \beta^{\prime}(\sigma)\left\|\eta_{x}(\sigma)\right\|^{2} d \sigma } \\
& {[1}
\end{aligned}
$$

First, we choose $\varepsilon_{1}$ small enough such that

$$
\begin{aligned}
d_{1}-2 \varepsilon_{1} & >0, \\
\frac{d_{2}}{4}-\frac{L}{2} \varepsilon_{1} & >0 .
\end{aligned}
$$

By setting

$$
\begin{aligned}
& \varepsilon_{2}=\frac{\left(d_{1}-2 \varepsilon_{1}\right) N_{1}}{2 N_{2}}, \\
& \varepsilon_{3}=\frac{2 c N_{3}}{N_{2}},
\end{aligned}
$$

we obtain

$$
\begin{aligned}
\mathscr{L}^{\prime}(t) \leq & -\left[\frac{1}{2}\left(d_{1}-2 \varepsilon_{1}\right) N_{1}\right] \int_{0}^{L}\left(u_{x}+\frac{1}{2} w_{x}^{2}\right)^{2} d x \\
& -\left[\frac{1}{2} N_{2}-N_{1}\right] \int_{0}^{L} u_{t}^{2} d x-\left[\left(\frac{d_{2}}{4}-\frac{L}{2} \varepsilon_{1}\right) N_{1}\right] \int_{0}^{L} w_{x x}^{2} d x \\
& -\left[C N-\frac{1}{2} N_{1}-\xi\right] \int_{0}^{L} \omega_{t}^{2} d x \\
& -\left[\widehat{c} N_{3}-\frac{\delta_{1}^{2}}{4 \varepsilon_{1}} N_{1}-c\left(1+\frac{N_{2}}{N_{1}}\right) N_{2}\right] \int_{0}^{L} \theta^{2} d
\end{aligned}
$$

$$
\begin{aligned}
& -\left[\widehat{r} N_{3}-\frac{\delta_{2}^{2}}{4 \varepsilon_{1}} N_{1}-c\left(1+\frac{N_{2}}{N_{1}}\right) N_{2}\right] \int_{0}^{L} P^{2} d x \\
& -\left[C N-c N_{1}+\eta_{1}\right] \int_{0}^{L} z^{2}(x, 1, t) d x-2 F_{4}(t) \\
& -\left[\frac{\alpha_{1}}{4} N-c N_{2}-\mu_{0} N_{3}\right]\|\eta\|_{\mathscr{M}_{\beta}}^{2} \\
& -\left[\frac{\alpha_{2}}{4} N-\lambda_{0} N_{3}\right]\|v\|_{\mathscr{M}_{\lambda}}^{2} \\
& +\left[\frac{1}{4} N-C_{\mu_{0}} N_{3}\right] \int_{0}^{\infty} \beta^{\prime}(\sigma)\left\|\eta_{x}(\sigma)\right\|^{2} d \sigma \\
& +\left[\frac{1}{4} N-C_{\lambda_{0}} N_{3}\right] \int_{0}^{\infty} \lambda^{\prime}(\sigma)\left\|v_{x}(\sigma)\right\|^{2} d \sigma .
\end{aligned}
$$

Next, we carefully choose our constants so that the terms inside the brackets are positive.

We choose $N_{2}$ large enough such that

$$
k_{1}=\frac{1}{2} N_{2}-N_{1}>0
$$

Then, we choose $N_{3}$ large enough such that

$$
\begin{aligned}
& k_{2}=\widehat{r} N_{3}-\frac{\delta_{2}^{2}}{4 \varepsilon_{1}} N_{1}-c\left(1+\frac{N_{2}}{N_{1}}\right) N_{2}>0, \\
& k_{3}=\widehat{r} N_{3}-\frac{\delta_{2}^{2}}{4 \varepsilon_{1}} N_{1}-c\left(1+\frac{N_{2}}{N_{1}}\right) N_{2}>0 .
\end{aligned}
$$

Thus, we arrive at

$$
\begin{aligned}
\mathscr{L}^{\prime}(t) \leq & -k_{0} \int_{0}^{L}\left(u_{x}+\frac{1}{2} w_{x}^{2}\right)^{2} d x-k_{1} \int_{0}^{L} u_{t}^{2} d x-k_{4} \int_{0}^{L} w_{x x}^{2} d x \\
& -(C N-c) \int_{0}^{L} \omega_{t}^{2} d x-k_{2} \int_{0}^{L} \theta^{2} d x-k_{3} \int_{0}^{L} P^{2} d x \\
& -(C N-c) \int_{0}^{L} z^{2}(x, 1, t) d x-2 F_{4}(t) \\
& -\left(\frac{\alpha_{1}}{4} N-c\right)\|\eta\|_{\mathscr{M}_{\beta}}^{2}-\left(\frac{\alpha_{2}}{4} N-c\right)\|v\|_{\mathscr{M}_{\lambda}}^{2} \\
& +\left(\frac{1}{4} N-c\right) \int_{0}^{\infty} \beta^{\prime}(\sigma)\left\|\eta_{x}(\sigma)\right\|^{2} d \sigma \\
& +\left(\frac{1}{4} N-c\right) \int_{0}^{\infty} \lambda^{\prime}(\sigma)\left\|v_{x}(\sigma)\right\|^{2} d \sigma
\end{aligned}
$$

where $k_{0}=(1 / 2)\left(d_{1}-2 \varepsilon_{1}\right) N_{1}$ and $k_{4}=\left(\left(d_{2} / 4\right)-(L / 2) \varepsilon_{1}\right) N_{1}$. On the other hand, we let

$$
\mathfrak{T}(t)=\sum_{i=1}^{i=3} N_{i} F_{i}(t)+F_{4}(t) .
$$


Exploiting Young's, Cauchy-Schwarz's, and Poincaré's inequalities, we get

$$
\begin{aligned}
|\mathfrak{T}(t)| \leq & c \int_{0}^{L}\left(\omega_{t}^{2}+u_{t}^{2}+\left(u_{x}+\frac{1}{2} w_{x}^{2}\right)^{2}+\omega_{x x}^{2}+\theta^{2}+P^{2}\right) d x \\
& +c\|\eta\|_{\mathscr{M}_{\beta}}^{2}+c\|v\|_{\mathscr{M}_{\lambda}}^{2}+c \int_{0}^{L} \int_{0}^{1} z^{2}(x, \rho, t) d \rho d x .
\end{aligned}
$$

Then,

$$
|\mathfrak{I}(t)| \leq c E(t)
$$

Consequently, we obtain

$$
|\mathfrak{I}(t)|=|\mathscr{L}(t)-N E(t)| \leq c E(t)
$$

that is,

$$
(N-c) E(t) \leq \mathscr{L}(t) \leq(N+c) E(t) .
$$

Now, we choose $N$ large enough such that

$$
\begin{aligned}
& N-c>0, \\
& \frac{\alpha_{1}}{4} N-c>0, \\
& \frac{\alpha_{2}}{4} N-c>0, \\
& \frac{1}{4} N-c>0>0, \\
& C N-c>0 .
\end{aligned}
$$
give

Exploiting (107), estimates (150) and (155), respectively,

$$
\mathscr{L}^{\prime}(t) \leq-a_{1} E(t)
$$

for some $a_{1}>0$, and

$$
c_{1} E(t) \leq \mathscr{L}(t) \leq c_{2} E(t), \quad \forall t \geq 0,
$$

for some $c_{1}, c_{2}>0$; we have

$$
\mathscr{L}(t) \sim E(t)
$$

A combination with (157) and (158) gives

$$
\mathscr{L}^{\prime}(t) \leq-C_{1} \mathscr{L}(t), \quad \forall t \geq 0,
$$

where $C_{1}=a_{1} / c_{2}$.

Finally, by simple integration of (159) and (160), we obtain the result (141).

\section{Data Availability}

No data were used to support the study.

\section{Conflicts of Interest}

This work does not have any conflicts of interest.

\section{Acknowledgments}

The fifth author extends their appreciation to the Deanship of Scientific Research at King Khalid University for funding this work through General Research Project under Grant No. G.R.P-10/42.

\section{References}

[1] A. S. Nicaise, C. Pignotti, and J. Valein, "Exponential stability of the wave equation with boundary time-varying delay," Discrete Contin. Dyn. Syst. Ser. S, vol. 2, pp. 559-581, 2009.

[2] A. Benabdallah and I. Lasiecka, "Exponential decay rates for a full von Kármán system of dynamic thermoelasticity," Journal of Differential Equations, vol. 160, pp. 51-93, 2000.

[3] A. Benabdallah and D. Teniou, "Exponential stability of a von Kármán model with thermal effects," Electron. J. Differ. Equations, vol. 7, p. 13, 1998.

[4] S. Boulaaras and N. Doudi, "Global existence and exponential stability of coupled Lamesystem with distributed delay and source term without memory term," Boundary Value Problems, vol. 2020, no. 1, 2020 .

[5] S. M. Boulaaras, A. Choucha, A. Zara, M. Abdalla, and B. B. Cheri, "Global existence and decay estimates of energy of solutions for a new class of -Laplacian heat equations with logarithmic nonlinearity," Journal of Function Spaces, vol. 2021, Article ID 5558818, 11 pages, 2021.

[6] L. Bouzettouta and A. Djebabla, "Exponential stabilization of the full von Kármán beam by a thermal effect and a frictional damping and distributed delay," Journal of Mathematical Physics, vol. 60, article 041506, 2019.

[7] A. Choucha and D. Ouchenane, "Local existence and blow up of solutions to a logarithmic nonlinear wave equation with time-varying delay," Studia. UBB. Math, 2020.

[8] A. Choucha, S. M. Boulaaras, D. Ouchenane, B. B. Cherif, and M. Abdalla, "Exponential stability of swelling porous elastic with a viscoelastic damping and distributed delay term," Journal of Function Spaces, vol. 2021, Article ID 5581634, 8 pages, 2021.

[9] A. Choucha, D. Ouchenane, and S. Boulaaras, "Well posedness and stability result for a thermoelastic laminated Timoshenko beam with distributed delay term," Mathematical Methods in the Applied Sciences, vol. 43, no. 17, pp. 9983-10004, 2020.

[10] I. Chueshov and I. Lasiecka, Von Kármán Evolution Equations, Springer Monographs in Mathematics, Springer, New York, 2010.

[11] C. M. Dafermos, "Asymptotic stability in viscoelasticity," Archive for Rational Mechanics and Analysis, vol. 37, no. 4, pp. 297-308, 1970.

[12] A. Djebabla and N. Tatar, "Exponential stabilization of the full von Kármán beam by a thermal effect and a frictional damping," Georgian Mathematical Journal, vol. 20, pp. 427-438, 2013. 
[13] A. Favini, M. Ann Horn, I. Lasiecka, and D. Tataru, "Global existence, uniqueness and regularity of solutions to a von Kármán system with nonlinear boundary dissipation," Differential and Integral Equations, vol. 9, pp. 267-294, 1996.

[14] T. Fastovska, "Upper semicontinuous attractor for 2D Mindlin-Timoshenko thermoelastic model with memory," Communications on Pure \& Applied Analysis, vol. 6, no. 1, pp. 83-101, 2007.

[15] A. E. Green and P. M. Naghdi, "On undamped heat waves in an elastic solid," Journal of Thermal Stresses, vol. 15, pp. 253264, 1992.

[16] M. A. Horn and I. Lasiecka, "Global stabilization of a dynamic von Kármán plate with nonlinear boundary feedback," Applied Mathematics and Optimization, vol. 31, pp. 57-84, 1995.

[17] M. Ann Horn and I. Lasiecka, "Uniform decay of weak solutions to a von Kármán plate with nonlinear boundary dissipation," Differential and Integral equations, vol. 7, pp. 885-908, 1994.

[18] J. U. Kim and Y. Renardy, "Boundary control of the Timoshenko beam," SIAM Journal on Control and Optimization, vol. 25, no. 6, pp. 1417-1429, 1987.

[19] M. Kirane, B. Said-houari, and M. N. Anwar, "Stability result for the Timoshenko system with a time-varying delay term in the internal feedbacks," Pure and Applied Analysis, vol. 10, no. 2, pp. 667-686, 2011.

[20] J. E. Lagnese, "Modelling and stabilization of nonlinear plates," in Estimation and Control of Distributed Parameter Systems. International Series of Numerical Mathematics / Internationale Schriftenreihe zur Numerischen Mathematik / Série Internationale d'Analyse Numérique, W. Desch, F. Kappel, and K. Kunisch, Eds., vol. 100, pp. 247-264, Birkhäuser, Basel, 1991.

[21] I. Lasiecka, "Uniform stabilizability of a full von Kármán system with nonlinear boundary feedback," SIAM Journal on Control and Optimization, vol. 36, pp. 1376-1422, 1998.

[22] I. Lasiecka, "Uniform decay rates for full von Kármán system of dynamic thermoelasticity with free boundary conditions and partial boundary dissipation," Communications in Partial Differential Equations, vol. 24, pp. 1801-1847, 1999.

[23] G. Perla Menzala, A. F. Pazoto, and E. Zuazua, "Stabilization of Berger-Timoshenko's equation as limit of the uniform stabilization of the von Kármán system of beams and plates," ESAIM: Mathematical Modelling and Numerical Analysis, vol. 36, pp. 657-691, 2002.

[24] G. P. Menzala and E. Zuazua, "Explicit exponential decay rates for solutions of von Kármán's system of thermoelastic plates," Comptes Rendus de l'Academie des Sciences-Serie I-Mathematique, vol. 324, pp. 49-54, 1997.

[25] J. P. Puel and M. Tucsnak, "Boundary stabilization for the von Kármán equations," SIAM Journal on Control and Optimization, vol. 33, pp. 255-273, 1995.

[26] Z. Quanxin, "Stabilization of stochastic nonlinear delay systems with exogenous disturbances and the event-triggered feedback control," IEEE Transactions on Automatic Control, vol. 64, no. 9, pp. 3764-3771, 2019.

[27] Z. Quanxin and H. Tingwen, "Stability analysis for a class of stochastic delay nonlinear systems driven by G-Brownian motion," Systems \& Control Letters, vol. 140, article 104699, 2020.

[28] M. A. Ragusa and A. Tachikawa, "Partial regularity of the minimizers of quadratic functionals with VMO coefficients," Jour- nal of the London Mathematical Society, vol. 72, no. 3, pp. 609620, 2005.

[29] M. Reissig and Y. G. Wang, "Cauchy problems for linear thermoelastic systems of type III in one space variable," Mathematicsl Methods in the Applied Sciences, vol. 28, no. 11, pp. 13591381, 2005.

[30] S. Timoshenko, "On the correction for shear of the differential equation for transverse vibrations of prismaticbars," Philosophical Magazine, vol. 41, pp. 744-746, 1921.

[31] A. Aouadi and A. Miranville, "Quasi-stability and global attractor in nonlinear thermoelastic diffusion plate with memory," Evolution Equations \& Control Theory, vol. 4, pp. 241263, 2015.

[32] A. Aouadi and A. Miranville, "Smooth attractor for a nonlinear thermoelastic diffusion thin plate based on GurtinPipkinâ $€^{\mathrm{TM}}$ s model," Asymptotic Analysis, vol. 95, pp. 129$160,2015$.

[33] M. Aouadi and A. Castejon, "Properties of global and exponential attractors for nonlinear thermo-diffusion Timoshenko system," Journal of Mathematical Physics, vol. 60, article 081503, 2019.

[34] S. Boulaaras, A. Choucha, B. Cherif et al., "Blow up of solutions for a system of two singular nonlocal viscoelastic equations with damping, general source terms and a wide class of relaxation functions," AIMS Mathematics, vol. 6, no. 5, pp. 46644676, 2021.

[35] A. Choucha, S. Boulaaras, D. Ouchenane, S. Alkhalaf, I. Mekawy, and M. Abdalla, "On the system of coupled nondegenerate Kirchhoff equations with distributed delay: global existence and exponential decay," Journal of Function Spaces, vol. 2021, Article ID 5577277, 13 pages, 2021.

[36] A. Menaceur, S. Boulaaras, A. Makhlouf, K. Rajagobal, and M. Abdalla, "Limit cycles of a class of perturbed differential systems via the first-order averaging method," Complexity, vol. 2021, Article ID 5581423, 6 pages, 2021.

[37] M. Aouadi, M. Campo, M. I. M. Copetti, and J. R. Fernández, "Existence, stability and numerical results for a Timoshenko beam with thermodiffusion effects," Zeitschrift für angewandte Mathematik und Physik, vol. 70, article 117, 2019.

[38] Y. Qin and X. Pan, "Global existence, asymptotic behavior and uniform attractors for a non- autonomous Timoshenko system of thermoelasticity of type III with a time- varying delay," Journal of Mathematical Analysis and Applications, vol. 484, no. 1, article 123672, 2020.

[39] C. Giorgi, M. G. Naso, and V. Pata, "Exponential stability in linear heat conduction with memory: a semigroup approach," Communications in Applied Analysis, vol. 5, pp. 121-134, 2001.

[40] T. Kato, "Linear and quasilinear equations of evolution of hyperbolic type,” C.I.M.E., II, 1976. 